\title{
LOCAL WELL-POSEDNESS FOR THE QUASI-LINEAR HAMILTONIAN SCHRÖDINGER EQUATION ON TORI
}

\author{
ROBERTO FEOLA AND FELICE IANDOLI
}

\begin{abstract}
We prove a local in time well-posedness result for quasi-linear Hamiltonian Schrödinger equations on $\mathbb{T}^{d}$ for any $d \geq 1$. For any initial condition in the Sobolev space $H^{s}$, with $s$ large, we prove the existence and uniqueness of classical solutions of the Cauchy problem associated to the equation. The lifespan of such a solution depends only on the size of the initial datum. Moreover we prove the continuity of the solution map.
\end{abstract}

\section{CONTENTS}

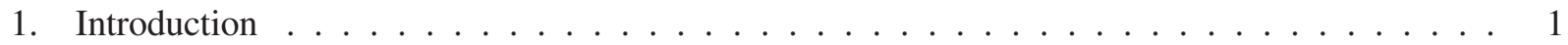

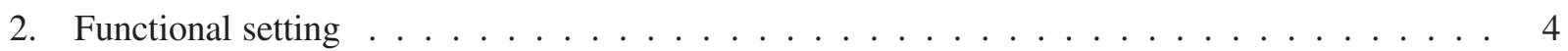

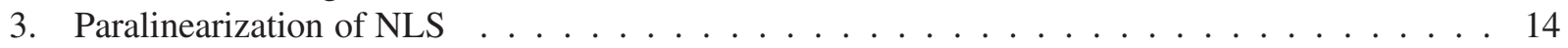

4. Basic energy estimates . . . . . . . . . . . . . . . . . . . 16

5. Proof of the main Theorem $1.2 \ldots \ldots \ldots \ldots \ldots \ldots \ldots \ldots$

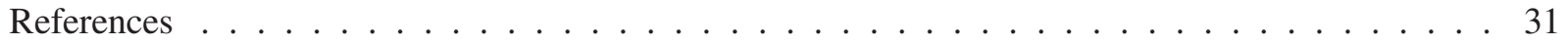

\section{INTRODUCTION}

In this paper we study the local in time solvability of the Cauchy problem associated to the following quasi-linear perturbation of the Schrödinger equation

$$
\mathrm{i} u_{t}-\Delta u+P(u)=0, \quad u=u(t, x), \quad x=\left(x_{1}, \ldots, x_{d}\right) \in \mathbb{T}^{d}:=(\mathbb{R} / 2 \pi \mathbb{Z})^{d}
$$

with

$$
P(u):=\left(\partial_{\bar{u}} F\right)(u, \nabla u)-\sum_{j=1}^{d} \partial_{x_{j}}\left(\partial_{\bar{u}_{x_{j}}} F\right)(u, \nabla u),
$$

where we denoted $\partial_{u}:=\left(\partial_{\operatorname{Re}(u)}-\mathrm{i} \partial_{\operatorname{Im}(u)}\right) / 2$ and $\partial_{\bar{u}}:=\left(\partial_{\operatorname{Re}(u)}+\mathrm{i} \partial_{\operatorname{Im}(u)}\right) / 2$ the Wirtinger derivatives. The function $F\left(y_{0}, y_{1}, \ldots, y_{d}\right)$ is in $C^{\infty}\left(\mathbb{C}^{d+1}, \mathbb{R}\right)$ in the real sense, i.e. $F$ is $C^{\infty}$ as function of $\operatorname{Re}\left(y_{i}\right)$, $\operatorname{Im}\left(y_{i}\right)$. Moreover we assume that $F$ has a zero of order at least 3 at the origin, so that $P$ has a zero of order at least 2 at the origin. Here $\nabla u=\left(\partial_{x_{1}} u, \ldots, \partial_{x_{d}} u\right)$ is the gradient and $\Delta$ denotes the Laplacian operator defined by linearity as

$$
\Delta e^{\mathrm{i} j \cdot x}=-|j|^{2} e^{\mathrm{i} j \cdot x}, \quad \forall j \in \mathbb{Z}^{d} .
$$

Notice that equation (1.1) is Hamiltonian, i.e.

$$
u_{t}=\mathrm{i} \nabla \bar{u} H(u, \bar{u}), \quad H(u, \bar{u}):=\int_{\mathbb{T}^{d}}|\nabla u|^{2}+F(u, \nabla u) d x,
$$

Key words and phrases. quasi-linear Schrödinger, Hamiltonian, para-differential calculus, energy estimates, wellposedness.

Felice Iandoli has been supported by ERC grant ANADEL 757996. Roberto Feola has been supported by the Centre Henri Lebesgue ANR-11-LABX- 0020-01 and by ANR-15-CE40-0001-02 "BEKAM" of the ANR. 
where $\nabla_{\bar{u}}:=\left(\nabla_{\operatorname{Re}(u)}-\mathrm{i} \nabla_{\operatorname{Im}(u)}\right) / 2$ and $\nabla_{\operatorname{Re}(u)}, \nabla_{\operatorname{Im}(u)}$ denote the $L^{2}$-gradient. In order to be able to consider initial data with big size we assume that the function $F$, defining the nonlinearity, satisfies following ellipticity condition.

Hypothesis 1.1. (Global ellipticity). We assume that there exist constants $\mathrm{c}_{1}, \mathrm{c}_{2}>0$ such that the following holds. For any $\xi=\left(\xi_{1}, \ldots, \xi_{d}\right) \in \mathbb{R}^{d}, y=\left(y_{0}, \ldots, y_{d}\right) \in \mathbb{C}^{d+1}$ one has

$$
\begin{gathered}
\sum_{j, k=1}^{d} \xi_{j} \xi_{k}\left(\delta_{j k}+\partial_{y_{j}} \partial_{\overline{y_{k}}} F(y)\right) \geq \mathrm{c}_{1}|\xi|^{2}, \\
\left(1+|\xi|^{-2} \sum_{j, k=1}^{d} \xi_{j} \xi_{k} \partial_{y_{j}} \partial_{\overline{y_{k}}} F(y)\right)^{2}-\left.\left.|| \xi\right|^{-2} \sum_{j, k=1}^{d} \xi_{j} \xi_{k} \partial_{\overline{y_{j}}} \partial_{\overline{y_{k}}} F(y)\right|^{2} \geq \mathrm{c}_{2},
\end{gathered}
$$

where $\delta_{j j}=1, \delta_{j k}=0$ for $j \neq k$.

The main result of this paper is the following.

Theorem 1.2. (Local well-posedness). Let $F$ be a function satisfying the Hypothesis 1.1 For any $s>$ $2 d+11$ the following holds true. Consider the equation (1.1) with initial condition $u(0, x)=u_{0}(x)$ in $H^{s}\left(\mathbb{T}^{d} ; \mathbb{C}\right)$, then there exists a time $0<T=T\left(\left\|u_{0}\right\|_{H^{s}}\right)$ and a unique solution

$$
u(t, x) \in C^{0}\left([0, T), H^{s}\left(\mathbb{T}^{d} ; \mathbb{C}\right)\right) \cap C^{1}\left([0, T), H^{s-2}\left(\mathbb{T}^{d} ; \mathbb{C}\right)\right) .
$$

Moreover the solution map $u_{0}(x) \mapsto u(t, x)$ is continuous with respect to the $H^{s}$ topology for any $t$ in $[0, T)$.

In the following we make some comments about the result we obtained.

- In the case of small initial conditions, i.e. $\left\|u_{0}\right\|_{H^{s}} \ll 1$, one can disregard the global ellipticity Hypothesis 1.1. Indeed for " $u$ small" the nonlinearity $F$ is always locally elliptic and one can prove the theorem in a similar way.

- When the initial condition satisfies $\left\|u_{0}\right\|_{H^{s}} \sim \varepsilon \ll 1$ it turns out that the life-span, implicit in this work, is $T \sim \varepsilon^{-1}$. We do not know if the solutions are globally in time defined or not. There are positive results when equation (1.1) is posed on $\mathbb{R}^{d}$. We refer, for instance, to [10] by De BouardHayashi-Saut and [11] by De Bouard-Hayashi-Naumkin-Saut. However in the latter papers the results are based on dispersive estimates which are not available on compact manifolds. Our result sets the stage for a refined normal form analysis to improve the time of existence, assuming certain non-resonance conditions. This is the content of the recent paper [15].

- Theorem 1.2 provides the well-posedness for the most general quasi-linear, Hamiltonian (local) nonlinearity. An example of physically relevant (see [22, 23, 16, 17]) quasi-linear Schrödinger is

$$
\mathrm{i} u_{t}-\Delta u+\left[\Delta\left(h\left(|u|^{2}\right)\right)\right] h^{\prime}\left(|u|^{2}\right) u-|u|^{2} u=0,
$$

for some real-valued $C^{\infty}$ function $h$. This model has been studied by many mathematicians, we refer for instance to [8] by Colin-Jeanjean and reference therein.

- We did not attempt to achieve the theorem in the best possible regularity $s$. We work in high regularity in order to perform suitable changes of coordinates and having a symbolic calculus at a sufficient order, which requires smoothness of the functions of the phase space.

- We prove the continuity of the solution map, we do not know if it is uniformly continuous or not. Unlike the semi-linear case (for which we refer to [5]), it is an hard problem to establish if the flow is more regular. These problems have been discussed in the paper [28] about Benjamin-Ono and related equations by Molinet-Saut-Tzvetkov. We also quote the survey article [33] by Tzvetkov.

To the best of our knowledge this theorem is the first of this kind on a compact manifold of dimension greater than or equal to 2 . For the same equation on the circle we quote our paper [12] and the one by BaldiHaus-Montalto [1]. In [1] a Nash-Moser iterative scheme has been used in order to obtain the existence 
of solutions in the case of small initial conditions. In our previous paper [12] we exploited the fact that in dimension one it is possible to conjugate the equation to constant coefficients by means of para-differential changes of coordinates. This technique has been used in several other papers to study the normal forms associated to these quasi-linear equations we quote for instance our papers [13, 14], and the earlier one by Berti-Delort [3] on the gravity-capillary water waves system. The proof we provide here is not based on this "reduction to constant coefficients" method which is peculiar of 1-dimensional problems. Furthermore we think that this proof, apart from being more general, is also simpler than the one given in [12].

The literature in the Euclidean space $\mathbb{R}^{d}$ is wider. After the 1-dimensional result by Poppenberg [30], there have been the pioneering works by Kenig-Ponce-Vega [19, 20, 21] in any dimension. More recently these results have been improved, in terms of regularity of the initial data, by Marzuola-Metcalfe-Tataru in $[24,25,26]$. We mention also that Chemin-Salort proved in [6] a very low regularity well-posedness for a particular quasi-linear Schrödinger equation in 3 dimensions coupled with an elliptic problem.

We make some short comments on the hypotheses we made on the equation. As already pointed out, the equation (1.1) is Hamiltonian. This is quite natural to assume when working on compact manifolds. On the Euclidean space one could make some milder assumptions because one could use the smoothing properties of the linear flow (proved by Constantin-Saut in [9]) to somewhat compensate the loss of derivatives introduced by the non Hamiltonian terms. These smoothing properties are not available on compact manifolds. Actually there are very interesting examples given by Christ in [7] of non Hamiltonian equations which are ill-posed on the circle $\mathbb{S}^{1}$ and well-posed on $\mathbb{R}$. Strictly speaking the Hamiltonian structure is not really fundamental for our method. For instance we could consider the not necessarily Hamiltonian nonlinearity

$$
P(u)=g(u) \Delta u+\mathrm{i} f(u) \cdot \nabla u+h(u),
$$

where $g: \mathbb{C} \rightarrow \mathbb{R}, f: \mathbb{C} \rightarrow \mathbb{R}^{d}, h: \mathbb{C} \rightarrow \mathbb{C}$ are smooth functions with a zero of order at least 2 . Our method would cover also this case. We did not insist on this fact because the equation above is morally Hamiltonian at the positive orders, in the sense that $f$ and $g$ are not linked, as in an Hamiltonian equation, but they enjoy the same reality properties of an Hamiltonian equation.

The Hypothesis 1.1 is needed in order to cover the case of large initial conditions, this is compatible with the global ellipticity condition we assumed in [12] and with the ones given in [20, 26]. As already said, this hypothesis is not necessary in the case of small data.

We discuss briefly the strategy of our proof. We begin by performing a para-linearization of the equation à la Bony [4] with respect to the variables $(u, \bar{u})$. Then, in the same spirit of [12], we construct the solutions of our problem by means of a quasi-linear iterative scheme à la Kato [18]. More precisely, starting from the para-linearized system, we build a sequence of linear problems which converges to a solution of the para-linearized system and hence to a solution of the original equation (1.1). At each step of the iteration one needs to solve a linear para-differential system, in the variable $(u, \bar{u})$, with non constant coefficients (see for instance (4.93)). This strategy is classical, one can find it for instance in the book by Metivier [27] for semilinear Schrödinger equations. Being our equation quasi-linear, we need some extra steps (w.r.t. [27]) in order to prove the existence of the solutions of the linear paradifferential system. We provide $a$ priori energy estimates (see Theorem 4.1). In order to do this, we decouple the equations for $(u, \bar{u})$ up to semilinear terms. This is done by applying changes of coordinates generated by para-differential operators. Once achieved such a diagonalization we are able to prove energy estimates in an energy-norm, which is equivalent to the Sobolev one. In constructing such energy norm, we need to introduce a microlocal cut-off (see (2.19)), relating the amplitude of the initial condition and the frequency of the solution, which allows us to obtain the result without any smallness assumption.

The paper is organized as follows. In Section 2 we give a short and self-contained introduction to the paradifferential calculus that is needed in the rest of the paper. In Section 3 we perform the para-linearization of the equation. In Section 4 we give an a priori energy estimate on the linearized equation by performing suitable changes of coordinates. In Section 5 we give the proof of Theorem 1.2 . 


\section{Functional SETting}

We denote by $H^{s}\left(\mathbb{T}^{d} ; \mathbb{C}\right)$ (respectively $H^{s}\left(\mathbb{T}^{d} ; \mathbb{C}^{2}\right)$ ) the usual Sobolev space of functions $\mathbb{T}^{d} \ni x \mapsto$ $u(x) \in \mathbb{C}\left(\right.$ resp. $\left.\mathbb{C}^{2}\right)$. We expand a function $u(x), x \in \mathbb{T}^{d}$, in Fourier series as

$$
u(x)=\frac{1}{(2 \pi)^{d / 2}} \sum_{n \in \mathbb{Z}^{d}} \widehat{u}(n) e^{\mathrm{i} n \cdot x}, \quad \widehat{u}(n):=\frac{1}{(2 \pi)^{d / 2}} \int_{\mathbb{T}^{d}} u(x) e^{-\mathrm{i} n \cdot x} d x .
$$

We also use the notation

$$
u_{n}^{+}:=u_{n}:=\widehat{u}(n) \quad \text { and } \quad u_{n}^{-}:=\overline{u_{n}}:=\overline{\widehat{u}(n)} .
$$

We set $\langle j\rangle:=\sqrt{1+|j|^{2}}$ for $j \in \mathbb{Z}^{d}$. We endow $H^{s}\left(\mathbb{T}^{d} ; \mathbb{C}\right)$ with the norm

$$
\|u(\cdot)\|_{H^{s}}^{2}:=\sum_{j \in \mathbb{Z}^{d}}\langle j\rangle^{2 s}\left|u_{j}\right|^{2} .
$$

For $U=\left(u_{1}, u_{2}\right) \in H^{s}\left(\mathbb{T}^{d} ; \mathbb{C}^{2}\right)$ we just set $\|U\|_{H^{s}}=\left\|u_{1}\right\|_{H^{s}}+\left\|u_{2}\right\|_{H^{s}}$. Moreover, for $r \in \mathbb{R}^{+}$, we denote by $B_{r}\left(H^{s}\left(\mathbb{T}^{d} ; \mathbb{C}\right)\right)\left(\right.$ resp. $\left.B_{r}\left(H^{s}\left(\mathbb{T}^{d} ; \mathbb{C}^{2}\right)\right)\right)$ the ball of $H^{s}\left(\mathbb{T}^{d} ; \mathbb{C}\right)\left(\right.$ resp. $\left.H^{s}\left(\mathbb{T}^{d} ; \mathbb{C}^{2}\right)\right)$ with radius $r$ centered at the origin. We shall also write the norm in (2.3) as

$$
\|u\|_{H^{s}}^{2}=\left(\langle D\rangle^{s} u,\langle D\rangle^{s} u\right)_{L^{2}}, \quad\langle D\rangle e^{\mathrm{i} j \cdot x}=\langle j\rangle e^{\mathrm{i} j \cdot x}, \quad \forall j \in \mathbb{Z}^{d},
$$

where $(\cdot, \cdot)_{L^{2}}$ denotes the standard complex $L^{2}$-scalar product

$$
(u, v)_{L^{2}}:=\int_{\mathbb{T}^{d}} u \bar{v} d x, \quad \forall u, v \in L^{2}\left(\mathbb{T}^{d} ; \mathbb{C}\right) .
$$

Notation. We shall use the notation $A \lesssim B$ to denote $A \leq C B$ where $C$ is a positive constant depending on parameters fixed once for all, for instance $d$ and $s$. We will emphasize by writing $\lesssim q$ when the constant $C$ depends on some other parameter $q$.

2.1. Basic Para-differential calculus. We introduce the symbols we shall use in this paper. We shall consider symbols $\mathbb{T}^{d} \times \mathbb{R}^{d} \ni(x, \xi) \rightarrow a(x, \xi)$ in the spaces $\mathcal{N}_{s}^{m}, m, s \in \mathbb{R}, s \geq 0$, defined by the norms

$$
|a|_{\mathcal{N}_{s}^{m}}:=\sup _{|\alpha|+|\beta| \leq s} \sup _{\xi \in \mathbb{R}^{d}}\langle\xi\rangle^{-m+|\beta|}\left\|\partial_{\xi}^{\beta} \partial_{x}^{\alpha} a(x, \xi)\right\|_{L^{\infty}} .
$$

The constant $m \in \mathbb{R}$ indicates the order of the symbols, while $s$ denotes its differentiability. Let $0<\epsilon<$ $1 / 4$, consider a smooth function $\chi: \mathbb{R} \rightarrow[0,1]$ satisfying

$$
\chi(\xi)= \begin{cases}1 & \text { if }|\xi| \leq 5 / 4 \\ 0 & \text { if }|\xi| \geq 8 / 5\end{cases}
$$

and define

$$
\chi_{\epsilon}(\xi):=\chi(|\xi| / \epsilon) .
$$

For a symbol $a(x, \xi)$ in $\mathcal{N}_{s}^{m}$ we define its (Weyl) quantization as

$$
T_{a} h:=\frac{1}{(2 \pi)^{d}} \sum_{j \in \mathbb{Z}^{d}} e^{\mathrm{i} j \cdot x} \sum_{k \in \mathbb{Z}^{d}} \chi_{\epsilon}\left(\frac{|j-k|}{\langle j+k\rangle}\right) \widehat{a}\left(j-k, \frac{j+k}{2}\right) \widehat{h}(k)
$$

where $\widehat{a}(\eta, \xi)$ denotes the $\eta$-Fourier coefficient of $a(x, \xi)$ in the variable $x \in \mathbb{T}^{d}$.

Remark 2.1. The definition of the operator $T_{a}$ is independent of the choice of the cut-off function $\chi_{\epsilon}$ up to regularizing operators (satisfying estimates of the form (2.18)), this will be one of the consequences of Lemma 2.3 
Remark 2.2. Let us consider a symbol $a(x, \xi)$ of order $m$ and set $A:=T_{a}$. Then one can check the following:

$$
\begin{aligned}
\bar{A}[h] & :=\overline{A[\bar{h}]}, \Rightarrow \quad \bar{A}=T_{\tilde{a}}, \quad \tilde{a}(x, \xi)=\overline{a(x,-\xi)} \\
\text { (Adjoint) }(A h, v)_{L^{2}} & =:\left(h, A^{*} v\right)_{L^{2}}, \quad \Rightarrow \quad A^{*}=T_{\bar{a}} .
\end{aligned}
$$

If the symbol a is real valued then the operator $T_{a}$ is self-adjoint with respect to the scalar product in (2.5).

Notation. Given a symbol $a(x, \xi)$ we shall also write

$$
T_{a}[\cdot]:=O p^{\mathrm{BW}}(a(x, \xi))[\cdot],
$$

to denote the associated para-differential operator.

We now recall some fundamental properties of para-differential operators.

Lemma 2.3. The following holds.

(i) Let $m_{1}, m_{2} \in \mathbb{R}, s>d / 2, s \in \mathbb{N}$ and $a \in \mathcal{N}_{s}^{m_{1}}, b \in \mathcal{N}_{s}^{m_{2}}$. One has

$$
|a b|_{\mathcal{N}_{s}^{m_{1}+m_{2}}}+|\{a, b\}|_{\mathcal{N}_{s-1}^{m_{1}+m_{2}-1}}+|\sigma(a, b)|_{\mathcal{N}_{s-2}^{m_{1}+m_{2}-2}} \lesssim|a|_{\mathcal{N}_{s}^{m_{1}}}|b|_{\mathcal{N}_{s}^{m_{2}}}
$$

where

$$
\begin{gathered}
\{a, b\}:=\sum_{j=1}^{d}\left(\left(\partial_{\xi_{j}} a\right)\left(\partial_{x_{j}} b\right)-\left(\partial_{x_{j}} a\right)\left(\partial_{\xi_{j}} b\right)\right) \\
\sigma(a, b):=\sum_{j, k=1}^{d}\left(\left(\partial_{\xi_{j} \xi_{k}} a\right)\left(\partial_{x_{j} x_{k}} b\right)-2\left(\partial_{x_{j} \xi_{k}} a\right)\left(\partial_{\xi_{j} x_{k}} b\right)+\left(\partial_{x_{j} x_{k}} a\right)\left(\partial_{\xi_{j} \xi_{k}} b\right)\right) .
\end{gathered}
$$

(ii) Let $s_{0}>d, s_{0} \in \mathbb{N}, m \in \mathbb{R}$ and $a \in \mathcal{N}_{s_{0}}^{m}$. Then, for any $s \in \mathbb{R}$, one has

$$
\left\|T_{a} h\right\|_{H^{s-m}} \lesssim|a|_{\mathcal{N}_{s_{0}}^{m}}\|h\|_{H^{s}}, \quad \forall h \in H^{s}\left(\mathbb{T}^{d} ; \mathbb{C}\right)
$$

(iii) Let $s_{0}>d, s_{0} \in \mathbb{N}, m \in \mathbb{R}, \rho \in \mathbb{N}$, and $a \in \mathcal{N}_{s_{0}+\rho}^{m}$. For $0<\epsilon_{2} \leq \epsilon_{1}<1 / 2$ and any $h \in H^{s}\left(\mathbb{T}^{d} ; \mathbb{C}\right)$, we define

$$
R_{a} h:=\frac{1}{(2 \pi)^{d}} \sum_{j \in \mathbb{Z}^{d}} e^{\mathrm{i} j \cdot x} \sum_{k \in \mathbb{Z}^{d}}\left(\chi_{\epsilon_{1}}-\chi_{\epsilon_{2}}\right)\left(\frac{|j-k|}{\langle j+k\rangle}\right) \widehat{a}\left(j-k, \frac{j+k}{2}\right) \widehat{h}(k),
$$

where $\chi_{\epsilon_{1}}, \chi_{\epsilon_{2}}$ are as in (2.8). Then one has

$$
\left\|R_{a} h\right\|_{H^{s+\rho-m}} \lesssim\|h\|_{H^{s}}|a|_{\mathcal{N}_{\rho+s_{0}}^{m}}, \quad \forall h \in H^{s}\left(\mathbb{T}^{d} ; \mathbb{C}\right) .
$$

(iv) Let $s_{0}>d, s_{0} \in \mathbb{N}, m \in \mathbb{R}$ and $a \in \mathcal{N}_{s_{0}}^{m}$. For $\mathrm{R}>0$, consider the cut-off function $\mathcal{X}_{\mathbb{R}} \in C^{\infty}\left(\mathbb{R}^{n} ; \mathbb{R}\right)$ defined as

$$
\mathcal{X}_{\mathrm{R}}(\xi):=1-\chi\left(\frac{|\xi|}{\mathrm{R}}\right)
$$

where $\chi$ is given in (2.7) and define the symbol $a_{\mathrm{R}}^{\perp}(x, \xi):=\left(1-\mathcal{X}_{\mathbb{R}}(\xi)\right) a(x, \xi)$. Then, for any $q \in \mathbb{R}$ such that $q+m \geq 0$, one has

$$
\left\|T_{a_{\mathrm{R}}^{\perp}} h\right\|_{H^{s+q}} \lesssim q, m \mathrm{R}^{q+m}\|h\|_{H^{s}}|a|_{\mathcal{N}_{s_{0}}^{m}}, \quad \forall h \in H^{s}\left(\mathbb{T}^{d} ; \mathbb{C}\right) .
$$

Proof. (i) For any $|\alpha|+|\beta| \leq s$ we have

$$
\partial_{x}^{\alpha} \partial_{\xi}^{\beta}(a(x, \xi) b(x, \xi))=\sum_{\substack{\alpha_{1}+\alpha_{2}=\alpha \\ \beta_{1}+\beta_{2}=\beta}} C_{\alpha, \beta}\left(\partial_{x}^{\alpha_{1}} \partial_{\xi}^{\beta_{1}} a\right)(x, \xi)\left(\partial_{x}^{\alpha_{2}} \partial_{\xi}^{\beta_{2}} b\right)(x, \xi)
$$

for some combinatoric coefficients $C_{\alpha, \beta}>0$. Then, recalling (2.6),

$$
\left\|\left(\partial_{x}^{\alpha_{1}} \partial_{\xi}^{\beta_{1}} a\right)(x, \xi)\left(\partial_{x}^{\alpha_{2}} \partial_{\xi}^{\beta_{2}} b\right)(x, \xi)\right\|_{L^{\infty}} \lesssim \alpha, \beta|a|_{\mathcal{N}_{s}^{m_{1}}}|b|_{\mathcal{N}_{s}^{m_{2}}}\langle\xi\rangle^{m_{1}+m_{2}-|\beta|} .
$$


This implies the (2.13) for the product $a b$. The (2.13) for the symbols $\{a, b\}$ and $\sigma(a, b)$ follows similarly using 2.14 and 2.15).

(ii) First of all notice that, since $a \in \mathcal{N}_{s_{0}}^{m}, s_{0} \in \mathbb{N}$, then (recall (2.6))

$$
\|a(\cdot, \xi)\|_{H^{s_{0}}} \lesssim\langle\xi\rangle^{m}|a|_{\mathcal{N}_{s_{0}}^{m}}, \quad \forall \xi \in \mathbb{Z}^{d}
$$

which implies

$$
|\widehat{a}(j, \xi)| \lesssim\langle\xi\rangle^{m}|a|_{\mathcal{N}_{s_{0}}^{m}}\langle j\rangle^{-s_{0}}, \quad \forall j, \xi \in \mathbb{Z}^{d} .
$$

Moreover, since $0<\epsilon<1 / 4$ we note that, for $\xi, \eta \in \mathbb{Z}^{d}$,

$$
\chi_{\epsilon}\left(\frac{|\xi-\eta|}{\langle\xi+\eta\rangle}\right) \neq 0 \Rightarrow\left\{\begin{array}{l}
(1-\tilde{\epsilon})|\xi| \leq(1+\tilde{\epsilon})|\eta| \\
(1-\tilde{\epsilon})|\eta| \leq(1+\tilde{\epsilon})|\xi|,
\end{array}\right.
$$

where $0<\tilde{\epsilon}<4 / 5$. Indeed, recalling (2.7)-(2.8), we have $|\xi| \leq\left(1+\frac{8}{5} \epsilon\right)|\eta|+\frac{8}{5} \epsilon|\xi|+\frac{8}{5} \epsilon$ which, for $|\eta| \neq 0$, implies $\left(1-\frac{8}{5} \epsilon\right)|\xi| \leq\left(1+\frac{16}{5} \epsilon\right)|\eta|$. This implies the first condition in (2.22) in the case $|\eta| \neq 0$. The case $|\eta|=0$ is trivial since the definition of the cut-off function $\chi_{\varepsilon}$ and $\varepsilon<1 / 4$ implies that $|\xi|=0$ as well. The second condition in (2.22) is similar. As a consequence we have $\langle\xi+\eta\rangle \sim\langle\xi\rangle$ : on one hand $|\xi+\eta| \leq|\xi|+|\eta| \leq(1+C)|\xi|$ for some $C=C(\tilde{\epsilon})>0$; on the other hand $|\xi|=\frac{1}{2}|\xi-\eta+\xi+\eta| \leq$ $\frac{1}{2}|\xi-\eta|+\frac{1}{2}|\xi+\eta| \leq \frac{1}{2} \frac{8 \epsilon}{5}\langle\xi+\eta\rangle+\frac{1}{2}\langle\xi+\eta\rangle$. Therefore, taking $s_{0}>d$,

$$
\begin{aligned}
\left\|T_{a} h\right\|_{H^{s-m}}^{2} & \stackrel{\sqrt[2.3]]{\lesssim}}{\lesssim} \sum_{\xi \in \mathbb{Z}^{d}}\langle\xi\rangle^{2(s-m)}\left|\sum_{\eta \in \mathbb{Z}^{d}} \chi_{\epsilon}\left(\frac{|\xi-\eta|}{\langle\xi+\eta\rangle}\right) \widehat{a}\left(\xi-\eta, \frac{\xi+\eta}{2}\right) \widehat{h}(\eta)\right|^{2} \\
& \lesssim \sum_{\xi \in \mathbb{Z}^{d}}\langle\xi\rangle^{-2 m}\left(\sum_{\eta \in \mathbb{Z}^{d}} \frac{\langle\xi\rangle^{m}}{\langle\xi-\eta\rangle^{s_{0}}}|\widehat{h}(\eta)|\langle\eta\rangle^{s}\right)^{2}|a|_{\mathcal{N}_{s_{0}}^{m}}^{2} \\
& \lesssim|a|_{\mathcal{N}_{s_{0}}^{m}}^{2} \sum_{\xi \in \mathbb{Z}^{d}}\left(\sum_{\eta \in \mathbb{Z}^{d}}\left|\widehat{h}(\eta)\langle\eta\rangle^{s} \frac{1}{\langle\xi-\eta\rangle^{s_{0}}}\right|\right)^{2} \\
& \lesssim|a|_{\mathcal{N}_{s_{0}}^{m}}^{2}\left\|\widehat{h}(\xi)\langle\xi\rangle^{s} \star\langle\xi\rangle^{-s_{0}}\right\|_{\ell^{2}\left(\mathbb{Z}^{d}\right)}^{2} \leq|a|_{\mathcal{N}_{s_{0}}^{m}}^{2}\left\|\widehat{h}(\xi)\langle\xi\rangle^{s}\right\|_{\ell^{2}\left(\mathbb{Z}^{d}\right)}^{2}\left\|\langle\xi\rangle^{-s_{0}}\right\|_{\ell^{1}\left(\mathbb{Z}^{d}\right)}^{2} \\
& \lesssim\|h\|_{H^{s}}^{2}|a|_{\mathcal{N}_{s_{0}}^{m}}^{2},
\end{aligned}
$$

where we denoted by $\star$ the convolution between sequences, in the penultimate passage we used the Young inequality for sequences and in the last one that $\langle\xi\rangle^{-s_{0}}$ is in $\ell^{1}\left(\mathbb{Z}^{d}\right)$ since $s_{0}>d$.

(iii) Notice that the set of $\xi, \eta$ such that $\left(\chi_{\epsilon_{1}}-\chi_{\epsilon_{2}}\right)(|\xi-\eta| /\langle\xi+\eta\rangle)=0$ contains the set such that

$$
|\xi-\eta| \geq \frac{8}{5} \epsilon_{1}\langle\xi+\eta\rangle \quad \text { or } \quad|\xi-\eta| \leq \frac{5}{4} \epsilon_{2}\langle\xi+\eta\rangle .
$$

Therefore $\left(\chi_{\epsilon_{1}}-\chi_{\epsilon_{2}}\right)(|\xi-\eta| /\langle\xi+\eta\rangle) \neq 0$ implies

$$
\frac{5}{4} \epsilon_{2}\langle\xi+\eta\rangle \leq|\xi-\eta| \leq \frac{8}{5} \epsilon_{1}\langle\xi+\eta\rangle .
$$

For $\xi \in \mathbb{Z}^{d}$ we denote $\mathcal{A}(\xi)$ the set of $\eta \in \mathbb{Z}^{d}$ such that the (2.24) holds. Moreover (reasoning as in (2.21), since $a \in \mathcal{N}_{s_{0}+\rho}^{m}$, we have that

$$
|\widehat{a}(j, \xi)| \lesssim\langle\xi\rangle^{m}|a|_{\mathcal{N}_{s_{0}+\rho}^{m}}\langle j\rangle^{-s_{0}-\rho}, \quad \forall j, \xi \in \mathbb{Z}^{d}
$$


To estimate the remainder in (2.17) we reason as in (2.23). By (2.24) and setting $\rho=s-s_{0}$ we have

$$
\begin{aligned}
\left\|R_{a} h\right\|_{H^{s+\rho-m}}^{2} & \stackrel{\sqrt{2.3}}{\lesssim} \sum_{\xi \in \mathbb{Z}^{d}}\langle\xi\rangle^{2(s+\rho-m)}\left|\left(\chi_{\epsilon_{1}}-\chi_{\epsilon_{2}}\right)\left(\frac{|\xi-\eta|}{\langle\xi+\eta\rangle}\right) \widehat{a}\left(\xi-\eta, \frac{\xi+\eta}{2}\right) \widehat{h}(\eta)\right|^{2} \\
& \stackrel{\substack{2.25 \\
\lesssim}}{\lesssim}\langle\xi\rangle^{-2 m}\left(\sum_{\xi \in \mathbb{Z}^{d}} \frac{\langle\xi-\eta\rangle^{\rho}\langle\xi+\eta\rangle^{m}}{\langle\xi-\eta\rangle^{\rho+s_{0}}}|\widehat{h}(\eta)|\langle\eta\rangle^{s}\right)^{2}|a|_{\mathcal{N}_{s_{0}+\rho}^{m}}^{2} \\
& \lesssim\left\|\widehat{h}(\xi)\langle\xi\rangle^{s} \star\langle\xi\rangle^{-s_{0}}\right\|_{\ell^{2}\left(\mathbb{Z}^{d}\right)}^{2}|a|_{\mathcal{N}_{\rho+s_{0}}^{m}}^{2} \lesssim\left\|\widehat{h}(\xi)\langle\xi\rangle^{s}\right\|_{\ell^{2}\left(\mathbb{Z}^{d}\right)}^{2}\left\|\langle\xi\rangle^{-s_{0}}\right\|_{\ell^{1}\left(\mathbb{Z}^{d}\right)}^{2}|a|_{\mathcal{N}_{\rho+s_{0}}^{m}}^{2} \\
& \lesssim\|h\|_{H^{s}}^{2}|a|_{\mathcal{N}_{\rho+s_{0}}^{m}}^{2},
\end{aligned}
$$

where we have denoted by $\star$ the convolution between sequences, in the penultimate step we used Young inequality for sequences, in the last one we used that $\langle\xi\rangle^{-s_{0}}$ is in $\ell^{1}\left(\mathbb{Z}^{d}\right)$ since $s_{0}>d$.

(iv) This item follows by reasoning exactly as in the proof of item (iii) and recalling that, by the definition of $\mathcal{X}_{\mathrm{R}}$ in 2.19), one has that $a_{\mathrm{R}}^{\perp}(x, \xi) \equiv 0$ for any $|\xi|>3 \mathrm{R}$.

Remark 2.4. The estimate (2.16) is not optimal. By following the more sophisticated proof by Metivier in [27] one could obtain the better bound with $|a|_{\mathcal{N}_{0}^{m}}$ instead of $|a|_{\mathcal{N}_{s_{0}}^{m}}$ on the right hand side.

Proposition 2.5. (Composition). Fix $s_{0}>d, s_{0} \in \mathbb{N}$, and $m_{1}, m_{2} \in \mathbb{R}$. Then the following holds.

(i) For $a \in \mathcal{N}_{s_{0}+4}^{m_{1}}$ and $b \in \mathcal{N}_{s_{0}+4}^{m_{2}}$ we have (recall (2.14), 2.15)

$$
T_{a} \circ T_{b}=T_{a b}+\frac{1}{2 \mathrm{i}} T_{\{a, b\}}-\frac{1}{8} T_{\sigma(a, b)}+R(a, b),
$$

where $R(a, b)$ is a remainder satisfying, for any $s \in \mathbb{R}$,

$$
\|R(a, b) h\|_{H^{s-m_{1}-m_{2}+3}} \lesssim\|h\|_{H^{s}}|a|_{\mathcal{N}_{s_{0}+4}^{m_{1}}}|b|_{\mathcal{N}_{s_{0}+4}^{m_{2}}} .
$$

Moreover, if $a, b \in H^{\rho+s_{0}}\left(\mathbb{T}^{d} ; \mathbb{C}\right)$ are functions (independent of $\xi \in \mathbb{R}^{n}$ ) then, $\forall s \in \mathbb{R}$,

$$
\left\|\left(T_{a} T_{b}-T_{a b}\right) h\right\|_{H^{s+\rho}} \lesssim\|h\|_{H^{s}}\|a\|_{H^{\rho+s_{0}}}\|b\|_{H^{\rho+s_{0}}} .
$$

(ii) Let $a, b$ as in item $(i)$ and, for $\mathrm{R}>0$, define $a_{\mathrm{R}}(x, \xi):=\mathcal{X}_{\mathrm{R}}(\xi) a(x, \xi), b_{\mathrm{R}}(x, \xi):=\mathcal{X}_{\mathrm{R}}(\xi) b(x, \xi)$ where $\mathcal{X}_{\mathrm{R}}(\xi)$ is defined in 2.19). Assume that $m_{1}+m_{2}-2 \leq 0$. Then

$$
T_{a_{\mathrm{R}}} \circ T_{b_{\mathrm{R}}}=T_{a_{\mathrm{R}} b_{\mathrm{R}}}+\frac{1}{2 \mathrm{i}} T_{\left\{a_{\mathrm{R}}, b_{\mathrm{R}}\right\}}-\frac{1}{8} T_{\sigma\left(a_{\mathrm{R}}, b_{\mathrm{R}}\right)}+R\left(a_{\mathrm{R}}, b_{\mathrm{R}}\right),
$$

where $R\left(a_{\mathrm{R}}, b_{\mathrm{R}}\right)$ is a remainder satisfying

$$
\left\|R\left(a_{\mathrm{R}}, b_{\mathrm{R}}\right) h\right\|_{H^{s-m_{1}-m_{2}+2}} \lesssim \mathrm{R}^{-1}\|h\|_{H^{s}}|a|_{\mathcal{N}_{s_{0}+4}^{m_{1}}}|b|_{\mathcal{N}_{s_{0}+4}^{m_{2}}} .
$$

Remark 2.6. We note that when applying the above proposition we consume four derivatives on the symbols. In the diagonalization procedure of Section 4 we shall apply this proposition several times. This requires a certain smoothness (in $x$ ) on the symbols. Since in our case the symbols will depend on the solution $u$ of (1.1), this smoothness is equivalent, thanks to the arguments in Section 2.3 to the regularity in $x$ of the solutions.

Proof of Proposition 2.5. We start by proving the 2.29). For $\xi, \theta, \eta \in \mathbb{Z}^{d}$ we define

$$
r_{1}(\xi, \theta, \eta):=\chi_{\epsilon}\left(\frac{|\xi-\theta|}{\langle\xi+\theta\rangle}\right) \chi_{\epsilon}\left(\frac{|\theta-\eta|}{\langle\theta+\eta\rangle}\right), \quad r_{2}(\xi, \eta):=\chi_{\epsilon}\left(\frac{|\xi-\eta|}{\langle\xi+\eta\rangle}\right) .
$$

Recalling (2.9) and that $a, b$ are functions we have

$$
\begin{aligned}
& R_{0} h:=\left(T_{a} T_{b}-T_{a b}\right) h, \\
& \widehat{\left(R_{0} h\right)}(\xi)=(2 \pi)^{-\frac{3 d}{2}} \sum_{\eta, \theta \in \mathbb{Z}^{d}}\left(r_{1}(\xi, \theta, \eta)-r_{2}(\xi, \eta)\right) \widehat{a}(\xi-\theta) \widehat{b}(\theta-\eta) \widehat{h}(\eta) .
\end{aligned}
$$


Let us define the sets

$$
\begin{aligned}
& D:=\left\{(\xi, \theta, \eta) \in \mathbb{Z}^{3 d}: r_{1}(\xi, \theta, \eta)-r_{2}(\xi, \eta)=0\right\} \\
& A:=\left\{(\xi, \theta, \eta) \in \mathbb{Z}^{3 d}: \frac{|\xi-\theta|}{\langle\xi+\theta\rangle} \leq \frac{5 \epsilon}{4}, \frac{|\xi-\eta|}{\langle\xi+\eta\rangle} \leq \frac{5 \epsilon}{4}, \frac{|\theta-\eta|}{\langle\theta+\eta\rangle} \leq \frac{5 \epsilon}{4}\right\}, \\
& B:=\left\{(\xi, \theta, \eta) \in \mathbb{Z}^{3 d}: \frac{|\xi-\theta|}{\langle\xi+\theta\rangle} \geq \frac{8 \epsilon}{5}, \frac{|\xi-\eta|}{\langle\xi+\eta\rangle} \geq \frac{8 \epsilon}{5}, \frac{|\theta-\eta|}{\langle\theta+\eta\rangle} \geq \frac{8 \epsilon}{5}\right\} .
\end{aligned}
$$

We note that

$$
D \supseteq A \cup B \quad \Rightarrow \quad D^{c} \subseteq A^{c} \cap B^{c}
$$

Let $(\xi, \theta, \eta) \in D^{c}$ and assume in particular that $(\xi, \theta, \eta) \in \operatorname{Supp}\left(r_{1}\right):=\overline{\left\{(\xi, \theta, \eta): r_{1} \neq 0\right\}}$. Then, reasoning as in (2.22), we can note that

$$
|\xi-\eta| \lesssim \epsilon\langle\xi+\eta\rangle \quad \text { and } \quad\langle\xi\rangle \sim\langle\eta\rangle
$$

Notice also that $(\xi, \theta, \eta) \in \operatorname{Supp}\left(r_{2}\right)$ implies the (2.37) as well. The rough idea of the proof is based on the fact that, if $(\xi, \theta, \eta) \in D^{c}$, then there are at least three equivalent frequencies among $\xi, \xi-\theta, \theta-\eta, \eta$, therefore (2.33) restricted to $(\xi, \theta, \eta) \in D^{c}$ is a regularizing operator. We need to estimate

$$
\left\|R_{0} h\right\|_{H^{s+\rho}}^{2} \lesssim \sum_{\xi \in \mathbb{Z}^{d}}\left(\sum_{\eta, \theta}^{*}|\widehat{a}(\xi-\theta)||\widehat{b}(\theta-\eta)||\widehat{h}(\eta)|\langle\xi\rangle^{s+\rho}\right)^{2}=I+I I+I I I
$$

where $\sum_{\eta, \theta}^{*}$ denotes the sum over indexes satisfying (2.37), the term $I$ denotes the sum on indexes satisfying also $|\xi-\theta|>c \epsilon|\xi|, I I$ denotes the sum on indexes satisfying also $|\eta-\theta|>c \epsilon|\eta|$, for some $0<c<1$ and $I I I$ is defined by difference. We estimate the term $I$. By using (2.37) and $|\xi-\theta|>c \epsilon|\xi|$ we get

$$
\begin{aligned}
I & \lesssim \sum_{\xi \in \mathbb{Z}^{d}}\left(\sum_{\eta, \theta}^{*}|\widehat{a}(\xi-\theta)||\widehat{b}(\theta-\eta)||\widehat{h}(\eta)|\langle\eta\rangle^{s}\langle\xi-\theta\rangle^{\rho}\right)^{2} \\
& \lesssim\left\||\widehat{h}(\xi)|\langle\xi\rangle^{s} \star|\widehat{a}(\xi)|\langle\xi\rangle^{\rho} \star|\widehat{b}(\xi)|\right\|_{\ell^{2}\left(\mathbb{Z}^{d}\right)}^{2} \\
& \lesssim\left\|\widehat{h}(\xi)\left|\langle\xi\rangle^{s}\left\|_{\ell^{2}\left(\mathbb{Z}^{d}\right)}^{2}\right\|\right| \widehat{a}(\xi)\left|\langle\xi\rangle^{\rho}\left\|_{\ell^{1}\left(\mathbb{Z}^{d}\right)}^{2}\right\| \widehat{b}(\xi)\right|\right\|_{\ell^{1}\left(\mathbb{Z}^{d}\right)}^{2} \\
& \lesssim\|h\|_{H^{s}}^{2}\|a\|_{H^{s_{0}+\rho}}^{2}\|b\|_{H^{s_{0}}}^{2},
\end{aligned}
$$

where in the last inequality we used Cauchy-Schwartz and $s_{0}>d>d / 2$.

Reasoning similarly one obtains $I I \lesssim\|h\|_{H^{s}}^{2}\|a\|_{H^{s_{0}}}^{2}\|b\|_{H^{s_{0}+\rho}}^{2}$. The sum $I I I$ is restricted to indexes satisfying (2.37) and $|\xi-\theta| \leq c \epsilon|\xi|,|\eta-\theta| \leq c \epsilon|\eta|$. For $0<c<1$ small enough these restrictions imply that $(\xi, \eta, \zeta) \in A$, which is a contradiction since $(\xi, \eta, \zeta) \in D^{c} \subseteq A^{c}$.

Let us check the (2.28). We first prove that

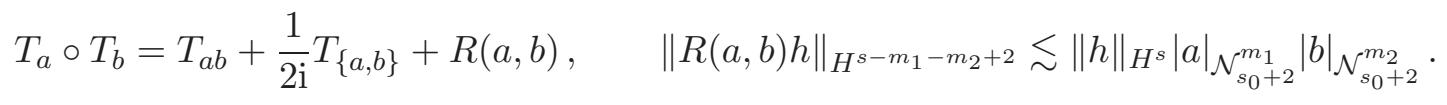


First of all we note that

$$
\begin{aligned}
\left(\widehat{T_{a} T_{b} h}\right)(\xi) & =\frac{1}{(\sqrt{2 \pi})^{3 d}} \sum_{\eta, \theta \in \mathbb{Z}^{d}} r_{1}(\xi, \theta, \eta) \widehat{a}\left(\xi-\theta, \frac{\xi+\theta}{2}\right) \widehat{b}\left(\theta-\eta, \frac{\theta+\eta}{2}\right) \widehat{h}(\eta), \\
\left.\widehat{\left(T_{a b} h\right.}\right)(\xi) & =\frac{1}{(\sqrt{2 \pi})^{3 d}} \sum_{\eta, \theta \in \mathbb{Z}^{d}} r_{2}(\xi, \eta) \widehat{a}\left(\xi-\theta, \frac{\xi+\eta}{2}\right) \widehat{b}\left(\theta-\eta, \frac{\xi+\eta}{2}\right) \widehat{h}(\eta), \\
\frac{1}{2 \mathrm{i}}\left(\widehat{T_{\{a, b\}} h}\right)(\xi) & =\frac{1}{2 \mathrm{i}(\sqrt{2 \pi})^{3 d}} \sum_{\eta, \theta \in \mathbb{Z}^{d}} r_{2}(\xi, \eta) \widehat{\left(\partial_{\xi} a\right)}\left(\xi-\theta, \frac{\xi+\eta}{2}\right) \cdot \widehat{\left(\partial_{x} b\right)}\left(\theta-\eta, \frac{\xi+\eta}{2}\right) \widehat{h}(\eta) \\
& -\frac{1}{2 \mathrm{i}(\sqrt{2 \pi})^{3 d}} \sum_{\eta, \theta \in \mathbb{Z}^{d}} r_{2}(\xi, \eta) \widehat{\left(\partial_{x} a\right)}\left(\xi-\theta, \frac{\xi+\eta}{2}\right) \cdot \widehat{\left(\partial_{\xi} b\right)}\left(\theta-\eta, \frac{\xi+\eta}{2}\right) \widehat{h}(\eta) .
\end{aligned}
$$

In the formulæ above we used the notation $\partial_{x}=\left(\partial_{x_{1}}, \ldots, \partial_{x_{d}}\right)$, similarly for $\partial_{\xi}$. We remark that we can substitute the cut-off function $r_{2}$ in 2.40, 2.41) with $r_{1}$ up to smoothing remainders. This follows because one can treat the cut-off function $r_{1}(\xi, \theta, \eta)-r_{2}(\xi, \eta)$ as done in the proof of (2.29). Write $\xi+\theta=$ $\xi+\eta+(\theta-\eta)$. By Taylor expanding the symbols at $\xi+\eta$, we have

$$
\begin{aligned}
\widehat{a}\left(\xi-\theta, \frac{\xi+\theta}{2}\right) & \left.=\widehat{a}\left(\xi-\theta, \frac{\xi+\eta}{2}\right)+\widehat{\left(\partial_{\xi} a\right.}\right)\left(\xi-\theta, \frac{\xi+\eta}{2}\right) \cdot \frac{\theta-\eta}{2} \\
& +\frac{1}{4} \sum_{j, k=1}^{d} \int_{0}^{1}(1-\sigma)\left(\widehat{\partial_{\xi_{j} \xi_{k}} a}\right)\left(\xi-\theta, \frac{\xi+\eta}{2}+\sigma \frac{\theta-\eta}{2}\right)\left(\theta_{j}-\eta_{j}\right)\left(\theta_{k}-\eta_{k}\right) d \sigma
\end{aligned}
$$

Similarly one obtains

$$
\begin{aligned}
\widehat{b}\left(\theta-\eta, \frac{\theta+\eta}{2}\right) & =\widehat{b}\left(\theta-\eta, \frac{\xi+\eta}{2}\right)+\widehat{\left(\partial_{\xi} b\right)}\left(\theta-\eta, \frac{\xi+\eta}{2}\right) \cdot \frac{\theta-\xi}{2} \\
& +\frac{1}{4} \sum_{j, k=1}^{d} \int_{0}^{1}(1-\sigma)\left(\widehat{\partial_{\xi_{j} \xi_{k}} b}\right)\left(\theta-\eta, \frac{\xi+\eta}{2}+\sigma \frac{\theta-\xi}{2}\right)\left(\theta_{j}-\xi_{j}\right)\left(\theta_{k}-\xi_{k}\right) d \sigma
\end{aligned}
$$

By (2.42), 2.43) we deduce that

$$
\begin{aligned}
& T_{a} T_{b} h-T_{a b} h-\frac{1}{2 \mathrm{i}} T_{\{a, b\}} h=\sum_{p=1}^{6} R_{p} h, \\
& \widehat{\left(R_{p} h\right)}(\xi):=\frac{1}{(\sqrt{2 \pi})^{3 d}} \sum_{\eta, \theta \in \mathbb{Z}^{d}} r_{1}(\xi, \theta, \eta) g_{p}(\xi, \theta, \eta) \widehat{h}(\eta),
\end{aligned}
$$

where the symbols $g_{i}$ are defined as

$$
\begin{aligned}
& g_{1}:=\frac{-1}{4} \sum_{j, k=1}^{d} \int_{0}^{1}(1-\sigma)\left(\widehat{\partial_{x_{k} x_{j}} a}\right)\left(\xi-\theta, \frac{\xi+\eta}{2}\right)\left(\widehat{\partial_{\xi_{k} \xi_{j}} b}\right)\left(\theta-\eta, \frac{\xi+\eta}{2}+\sigma \frac{\theta-\xi}{2}\right) d \sigma \\
& g_{2}:=\frac{-1}{4} \sum_{j, k=1}^{d} \int_{0}^{1}(1-\sigma)\left(\widehat{\partial_{\xi_{k} \xi_{j}} a}\right)\left(\xi-\theta, \frac{\xi+\eta}{2}+\sigma \frac{\theta-\eta}{2}\right)\left(\widehat{\partial_{x_{k} x_{j}} b}\right)\left(\theta-\eta, \frac{\xi+\eta}{2}\right) d \sigma, \\
& g_{3}:=\frac{1}{4} \sum_{j, k=1}^{d}\left(\widehat{\partial_{x_{j} \partial_{\xi_{k}}} a}\right)\left(\xi-\theta, \frac{\xi+\eta}{2}\right)\left(\widehat{\partial_{x_{k} \partial_{\xi_{j}}} b}\right)\left(\theta-\eta, \frac{\xi+\eta}{2}\right),
\end{aligned}
$$




$$
\begin{aligned}
& \left.g_{4}:=\frac{-1}{8 \mathrm{i}} \sum_{j, k, p=1}^{d} \int_{0}^{1}(1-\sigma)\left(\widehat{\partial_{x_{k} x_{j} \xi_{p}}} a\right)\left(\xi-\theta, \frac{\xi+\eta}{2}\right) \widehat{\left(\partial_{x_{p} \xi_{k} \xi_{j}}\right.} b\right)\left(\theta-\eta, \frac{\xi+\eta}{2}+\sigma \frac{\theta-\xi}{2}\right) d \sigma, \\
& g_{5}:=\frac{-1}{8 \mathrm{i}} \sum_{j, k, p=1}^{d} \int_{0}^{1}(1-\sigma)\left(\widehat{\partial_{\xi_{k} \xi_{j} x_{p}}} a\right)\left(\xi-\theta, \frac{\xi+\eta}{2}+\sigma \frac{\theta-\eta}{2}\right)\left(\widehat{\partial_{\xi_{p} x_{k} x_{j}}} b\right)\left(\theta-\eta, \frac{\xi+\eta}{2}\right) d \sigma, \\
& g_{6}:=\frac{1}{16} \sum_{j, k, p, q=1}^{d} \iint_{0}^{1}\left(1-\sigma_{1}\right)\left(1-\sigma_{2}\right)\left(\widehat{\partial_{\xi j}} \widehat{\xi_{k} x_{p} x_{q}} a\right)\left(\xi-\theta, \frac{\xi+\eta}{2}+\sigma_{1} \frac{\theta-\eta}{2}\right), \\
& \times\left(\widehat{\partial_{\xi_{p} \xi_{q} x_{j} x_{k}}} b\right)\left(\theta-\eta, \frac{\xi+\eta}{2}+\sigma_{2} \frac{\theta-\xi}{2}\right) d \sigma_{1} d \sigma_{2} .
\end{aligned}
$$

We prove the estimate (2.28) on each term of the sum in (2.44). First of all we note that $r_{1}(\xi, \theta, \eta) \neq 0$ implies that

$$
(\theta, \eta) \in\left\{\frac{|\xi-\theta|}{\langle\xi+\theta\rangle} \leq \frac{8}{5} \epsilon\right\} \bigcap\left\{\frac{|\theta-\eta|}{\langle\theta+\eta\rangle} \leq \frac{8}{5} \epsilon\right\}=: \mathcal{B}(\xi), \quad \xi \in \mathbb{Z}^{d}
$$

Moreover we note that

$$
(\theta, \eta) \in \mathcal{B}(\xi) \Rightarrow|\xi| \lesssim|\theta|,|\theta| \lesssim|\eta|,|\eta| \lesssim|\xi|
$$

We now study the term $R_{3} h$ in (2.44) depending on $g_{3}(\xi, \theta, \eta)$ in (2.47). We need to bound from above, for any $j, k=1, \ldots, d$, the $H^{s-m_{1}-m_{2}+2}$-Sobolev norm (see (2.51) $)$ of function $F_{j, k}(x)$ whose $\xi$-th Fourier coefficient is

$$
\begin{aligned}
\widehat{F}_{j, k}(\xi) & \left.:=\sum_{(\theta, \eta) \in \mathcal{B}(\xi)}\left(\widehat{\partial_{x_{j}} \partial_{\xi_{k}}} a\right)\left(\xi-\theta, \frac{\xi+\eta}{2}\right) \widehat{\left(\partial_{x_{k}} \partial_{\xi_{j}}\right.} b\right)\left(\theta-\eta, \frac{\xi+\eta}{2}\right) \widehat{h}(\eta) \\
& =\sum_{\eta \in \mathbb{Z}^{d}} \widehat{c_{j, k}}\left(\xi-\eta, \frac{\xi+\eta}{2}\right) \widehat{h}(\eta)
\end{aligned}
$$

where we have defined

$$
\begin{aligned}
\widehat{c_{j, k}}(p, \zeta) & :=\sum_{\ell \in \mathbb{Z}^{d}}\left(\widehat{\partial_{x_{j}} \partial_{\xi_{k}}} a\right)(p-\ell, \zeta)\left(\widehat{\partial_{x_{k}} \partial_{\xi_{j}}} b\right)(\ell, \zeta) 1_{\mathcal{C}(p, \zeta)}, \quad p, \zeta \in \mathbb{Z}^{d}, \\
\mathcal{C}(p, \zeta) & :=\left\{\ell \in \mathbb{Z}^{d}: \frac{|p-\ell|}{\langle 2 \zeta+\ell\rangle} \leq \frac{8}{5} \epsilon\right\} \bigcap\left\{\ell \in \mathbb{Z}^{d}: \frac{|\ell|}{\langle\ell-p+2 \zeta\rangle} \leq \frac{8}{5} \epsilon\right\}
\end{aligned}
$$

and $1_{\mathcal{C}(p, \zeta)}$ is the characteristic function of the set $\mathcal{C}(p, \zeta)$. Reasoning as in 2.52 , we can deduce that for $\ell \in \mathcal{C}(p, \zeta)$ one has

$$
|2 \zeta| \lesssim \frac{1}{2}|2 \zeta+p|
$$

Indeed $\ell \in \mathcal{C}(p, \zeta)$ implies $(\theta, \eta) \in \mathcal{B}(\xi)$ by setting

$$
2 \xi=2 \zeta+p, \quad 2 \theta=2 \ell+2 \zeta-p, \quad 2 \eta=2 \zeta-p .
$$

Hence the (2.54) follows by (2.52) by observing that $2 \zeta=\xi+\eta$. Using that $a \in \mathcal{N}_{s_{0}+4}^{m_{1}}, b \in \mathcal{N}_{s_{0}+4}^{m_{2}}$ and reasoning as in 2.21) we deduce

$$
\left|\widehat{c_{j, k}}(p, \zeta)\right| \lesssim\langle\zeta\rangle^{m_{1}+m_{2}-2}\langle p\rangle^{-s_{0}}|a|_{\mathcal{N}_{s_{0}+2}^{m_{1}}}|b|_{\mathcal{N}_{s_{0}+2}^{m_{2}}}
$$


By (2.53), 2.52), 2.3, we get

$$
\begin{aligned}
& \left\|F_{j, k}\right\|_{H^{s-m_{1}-m_{2}+2}}^{2} \lesssim \sum_{\xi \in \mathbb{Z}^{d}}\langle\xi\rangle^{-2 m_{1}-2 m_{2}+2}\left(\sum_{\eta \in \mathbb{Z}^{d}}\left|\widehat{c_{j, k}}\left(\xi-\eta, \frac{\xi+\eta}{2}\right)\right||\widehat{h}(\eta)|\langle\eta\rangle^{s}\right)^{2}
\end{aligned}
$$

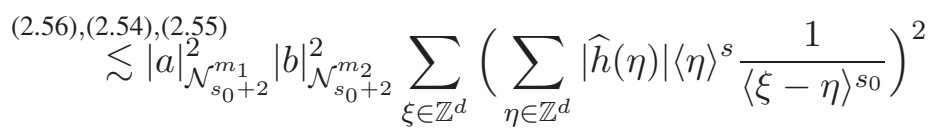

$$
\begin{aligned}
& \lesssim|a|_{\mathcal{N}_{s_{0}+2}^{m_{1}}}^{2}|b|_{\mathcal{N}_{s_{0}+2}^{m_{2}}}^{2}\left\|\widehat{h}(\xi) \mid\langle\xi\rangle^{s} \star\langle\xi\rangle^{-s_{0}}\right\|_{\ell^{2}\left(\mathbb{Z}^{d}\right)} \\
& \lesssim\|h\|_{H^{s}}^{2}|a|_{\mathcal{N}_{s_{0}+2}^{m_{1}}}^{2}|b|_{\mathcal{N}_{s_{0}+2}^{m_{2}}}^{2},
\end{aligned}
$$

where in the last step we used Young inequality for sequences, the Cauchy-Schwartz inequality and that $\langle\xi\rangle^{-s_{0}}$ is in $\ell^{1}\left(\mathbb{Z}^{d}\right)$ if $s_{0}>d$. Since the estimate above holds for any $j, k=1, \ldots, d$, we deduce the (2.38) for the remainder $R_{3} h$ in (2.44). By reasoning in the same way one can show that the remainders depending on $g_{1}, g_{2}$ in (2.45), (2.46) satisfy the bound in 2.38) and that the remainders $R_{p} h$ with $p=4,5,6$, satisfy the (2.28). In order to obtain the expansion (2.27) one can note that (see (2.45)

$$
\begin{aligned}
g_{1} & =-\frac{1}{8} \sum_{j, k=1}^{d}\left(\widehat{\partial_{x_{k} x_{j}} a}\right)\left(\xi-\theta, \frac{\xi+\eta}{2}\right)\left(\widehat{\partial_{\xi_{k} \xi_{j}} b}\right)\left(\theta-\eta, \frac{\xi+\eta}{2}\right) \\
& -\frac{1}{16} \sum_{j, k, p=1}^{d} \int_{0}^{1}(1-\sigma)^{2}\left(\widehat{\partial_{x_{k} x_{j}} a}\right)\left(\xi-\theta, \frac{\xi+\eta}{2}\right)\left(\widehat{\partial_{\xi_{k} \xi_{j} \xi_{p}}}\right)\left(\theta-\eta, \frac{\xi+\eta}{2}+\sigma \frac{\theta-\xi}{2}\right)\left(\theta_{p}-\xi_{p}\right) d \sigma,
\end{aligned}
$$

here we have used the identity $\int_{0}^{1} f(\sigma)(1-\sigma) d \sigma=\frac{1}{2} f(0)+\frac{1}{2} \int_{0}^{1} f^{\prime}(\tau)(1-\tau)^{2} d \tau$, which follows from the fundamental theorem of calculus. Expanding similarly the term $g_{2}$ in (2.46) and recalling the formula (2.15) one gets the 2.27). The estimate for the operator associated to the second summand in 2.57) follows by reasoning as done for the term in (2.53). This concludes the proof of item $(i)$. Item $(i i)$ follows by reasoning as before on the symbols $a_{\mathrm{R}}, b_{\mathrm{R}}$. Notice that the remainder $R\left(a_{\mathrm{R}}, b_{\mathrm{R}}\right)$ (see (2.28) ) maps $H^{s}$ to $H^{s-m_{1}-m_{2}+3}$. Actually using that $a_{\mathrm{R}} \equiv b_{\mathrm{R}} \equiv 0$ if $|\xi| \leq 3 \mathrm{R}$ one gets the 2.31).

Lemma 2.7. (Paraproduct). Fix $s_{0}>d / 2$ and let $f, g \in H^{s}(\mathbb{T} ; \mathbb{C})$ for $s \geq s_{0}$. Then

$$
f g=T_{f} g+T_{g} f+\mathcal{R}(f, g)
$$

where

$$
\widehat{\mathcal{R}(f, g)}(\xi)=\frac{1}{(2 \pi)^{d}} \sum_{\eta \in \mathbb{Z}^{d}} a(\xi-\eta, \xi) \widehat{f}(\xi-\eta) \widehat{g}(\eta), \quad|a(v, w)| \lesssim \frac{(1+\min (|v|,|w|))^{\rho}}{\left(1+\max (|v|,|w|)^{\rho}\right.},
$$

for any $\rho \geq 0$. For $0 \leq \rho \leq s-s_{0}$ one has

$$
\|\mathcal{R}(f, g)\|_{H^{s+\rho}} \lesssim\|f\|_{H^{s}}\|g\|_{H^{s}} .
$$

Proof. Notice that

$$
\widehat{(f g)}(\xi)=\sum_{\eta \in \mathbb{Z}^{d}} \widehat{f}(\xi-\eta) \widehat{g}(\eta)
$$

Consider the cut-off function $\chi_{\epsilon}$ defined in (2.8) and define a new cut-off function $\Theta: \mathbb{R} \rightarrow[0,1]$ as

$$
1=\chi_{\epsilon}\left(\frac{|\xi-\eta|}{\langle\xi+\eta\rangle}\right)+\chi_{\epsilon}\left(\frac{|\eta|}{\langle 2 \xi-\eta\rangle}\right)+\Theta(\xi, \eta)
$$

Recalling (2.61) and (2.9) we note that

$$
\widehat{\left(T_{f} g\right)}(\xi)=\sum_{\eta \in \mathbb{Z}^{d}} \chi_{\epsilon}\left(\frac{|\xi-\eta|}{\langle\xi+\eta\rangle}\right) \widehat{f}(\xi-\eta) \widehat{g}(\eta), \quad \widehat{\left(T_{g} f\right)}(\xi)=\sum_{\eta \in \mathbb{Z}^{d}} \chi_{\epsilon}\left(\frac{|\eta|}{\langle 2 \xi-\eta\rangle}\right) \widehat{f}(\xi-\eta) \widehat{g}(\eta),
$$


and

$$
\mathcal{R}:=\mathcal{R}(f, g), \quad \widehat{\mathcal{R}}(\xi):=\sum_{\eta \in \mathbb{Z}^{d}} \Theta(\xi, \eta) \widehat{f}(\xi-\eta) \widehat{g}(\eta) .
$$

To obtain the second in (2.63) one has to use the (2.9) and perform the change of variable $\xi-\eta \rightsquigarrow \eta$. By the definition of the cut-off function $\Theta(\xi, \eta)$ we deduce that, if $\Theta(\xi, \eta) \neq 0$ we must have

$$
|\xi-\eta| \geq \frac{5 \epsilon}{4}\langle\xi+\eta\rangle \quad \text { and } \quad|\eta| \geq \frac{5 \epsilon}{4}\langle 2 \xi-\eta\rangle \quad \Rightarrow \quad\langle\eta\rangle \sim\langle\xi-\eta\rangle .
$$

This implies that, setting $a(\xi-\eta, \eta):=\Theta(\xi, \eta)$, we get the (2.59). The (2.65) also implies that $\langle\xi\rangle \lesssim$ $\max \{\langle\xi-\eta\rangle,\langle\eta\rangle\}$. Then we have

$$
\begin{aligned}
\|\mathcal{R} h\|_{H^{s+\rho}}^{2} & \lesssim \sum_{\xi \in \mathbb{Z}^{d}}\left(\sum_{\eta \in \mathbb{Z}^{d}}|a(\xi-\eta, \eta)\|\widehat{f}(\xi-\eta)\| \widehat{g}(\eta)|\langle\xi\rangle^{s+\rho}\right)^{2} \\
& \stackrel{\lesssim}{\lesssim} \sum_{\xi \in \mathbb{Z}^{d}}\left(\sum_{\langle\xi-\eta\rangle \geq\langle\eta\rangle}\langle\xi-\eta\rangle^{s}|\widehat{f}(\xi-\eta)|\langle\eta\rangle^{\rho} \mid \widehat{g}(\eta)\right)^{2} \\
& +\sum_{\xi \in \mathbb{Z}^{d}}\left(\sum_{\langle\xi-\eta\rangle \leq\langle\eta\rangle}\langle\xi-\eta\rangle^{\rho}|\widehat{f}(\xi-\eta)||\widehat{g}(\eta)|\langle\eta\rangle^{s}\right)^{2} \\
& \lesssim \sum_{\xi, \eta \in \mathbb{Z}^{d}}\langle\eta\rangle^{2\left(s_{0}+\rho\right)}|\widehat{g}(\eta)|^{2}\langle\xi-\eta\rangle^{2 s}|\widehat{f}(\xi-\eta)|^{2} \\
& +\sum_{\xi, \eta \in \mathbb{Z}^{d}}\langle\eta\rangle^{2 s}|\widehat{g}(\eta)|^{2}\langle\xi-\eta\rangle^{2\left(s_{0}+\rho\right)}|\widehat{f}(\xi-\eta)|^{2} \\
& \lesssim\|f\|_{H^{s}}^{2}\|g\|_{H^{s_{0}}+\rho}^{2}+\|f\|_{H^{s_{0}}+\rho}^{2}\|g\|_{H^{s}}^{2}
\end{aligned}
$$

which implies the (2.60) for $s_{0}+\rho \leq s$.

2.2. Real-to-real, Self-adjoint operators. In this section we analyze some algebraic properties of paradifferential operators. Let us consider a linear operator

$$
M:=\left(M_{\sigma}^{\sigma^{\prime}}\right)_{\sigma, \sigma^{\prime} \in\{ \pm\}}:=:\left(\begin{array}{ll}
M_{+}^{+} & M_{+}^{-} \\
M_{-}^{+} & M_{-}^{-}
\end{array}\right): H^{s+p}\left(\mathbb{T}^{d} ; \mathbb{C}^{2}\right) \rightarrow H^{s}\left(\mathbb{T}^{d} ; \mathbb{C}^{2}\right)
$$

for some $p \in \mathbb{R}$. We have the following definition.

Definition 2.8. (Real-to-real maps). Consider a linear operator $A: H^{s+p}\left(\mathbb{T}^{d} ; \mathbb{C}\right) \rightarrow H^{s}\left(\mathbb{T}^{d} ; \mathbb{C}\right)$ for some $p \in \mathbb{R}$. We associate the linear operator $\bar{A}[\cdot]$ defined by the relation

$$
\bar{A}[v]:=\overline{A[\bar{v}]}, \quad \forall v \in H^{s+p}\left(\mathbb{T}^{d} ; \mathbb{C}\right) .
$$

We say that a matrix $M$ of operators acting in $\mathbb{C}^{2}$ of the form (2.66) is real-to-real, if it has the form

$$
M=\left(M_{\sigma}^{\sigma^{\prime}}\right)_{\sigma, \sigma^{\prime} \in\{ \pm\}}, \quad M_{\sigma}^{\sigma^{\prime}}=\overline{M_{-\sigma}^{-\sigma^{\prime}}}
$$

where $\overline{M_{\sigma}^{\sigma^{\prime}}}$ are defined as in (2.67).

Remark 2.9. Let $\mathfrak{F}$ a matrix of operators as in (2.66). If $\mathfrak{F}$ is real-to-real (according to Def. 2.8) then it preserves the subspace $\mathcal{U}$ defined as

$$
\mathcal{U}:=\left\{\left(u^{+}, u^{-}\right) \in L^{2}\left(\mathbb{T}^{d} ; \mathbb{C}\right) \times L^{2}\left(\mathbb{T}^{d} ; \mathbb{C}\right): u^{+}=\overline{u^{-}}\right\} .
$$

In particular it has the form (see (2.67), (2.68)

$$
\mathfrak{F}:=\left(\frac{A}{B} \frac{B}{A}\right) .
$$


We consider the scalar product on $L^{2}\left(\mathbb{T}^{d} ; \mathbb{C}^{2}\right) \cap \mathcal{U}$ (see (2.69) given by

$$
(U, V)_{L^{2}}:=\int_{\mathbb{T}^{d}} U \cdot \bar{V} d x:=\int_{\mathbb{T}^{d}}(u \bar{v}+\bar{u} v) d x, \quad U=\left[\frac{u}{u}\right], \quad V=\left[\frac{v}{v}\right] .
$$

We denote by $\mathfrak{F}^{*}$ its adjoint with respect to the scalar product 2.71

$$
(\mathfrak{F} U, V)_{L^{2}}=\left(U, \mathfrak{F}^{*} V\right)_{L^{2}}, \quad \forall U, V \in L^{2}\left(\mathbb{T}^{d} ; \mathbb{C}^{2}\right) \cap \mathcal{U}, \quad \mathfrak{F}^{*}:=\left(\begin{array}{ll}
A^{*} & \bar{B}^{*} \\
B^{*} & \bar{A}^{*}
\end{array}\right),
$$

where $A^{*}$ and $B^{*}$ are respectively the adjoints of the operators $A$ and $B$ with respect to the complex scalar product on $L^{2}\left(\mathbb{T}^{d} ; \mathbb{C}\right)$ in $(2.5)$.

Definition 2.10. (Self-adjointness). An operator $\mathfrak{F}$ of the form (2.70) is self-adjoint if and only if

$$
A^{*}=A, \quad \bar{B}=B^{*} .
$$

Remark 2.11. (Matrices of symbols). Recall Remark 2.2 Consider two symbols $a_{1}, a_{2} \in \mathcal{N}_{s}^{m}$ and the matrix

Define the operator (recall (2.12)

$$
A:=A(x, \xi):=\left(\frac{a_{1}(x, \xi)}{a_{2}(x,-\xi)} \frac{a_{2}(x, \xi)}{a_{1}(x,-\xi)}\right) .
$$

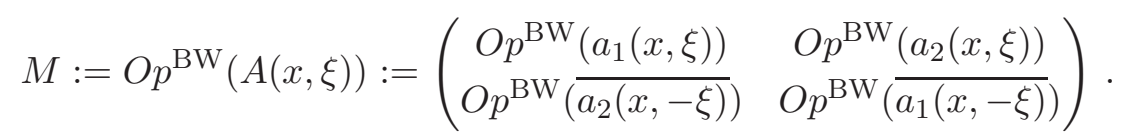

Recalling 2.10), 2.11), one can note that $M$ is real-to-real. Moreover $M$ is self-adjoint if and only if

$$
a_{1}(x, \xi)=\overline{a_{1}(x, \xi)}, \quad a_{2}(x,-\xi)=a_{2}(x, \xi) .
$$

2.3. Non-homogeneous symbols. In this section we study some properties of symbols depending nonlinearly on some function $u \in H^{s}\left(\mathbb{T}^{d} ; \mathbb{C}\right)$. We recall classical tame estimates for composition of functions (see for instance [29], [31], [32]). A function $f: \mathbb{T}^{d} \times B_{R} \rightarrow \mathbb{C}$, where $B_{R}:=\left\{y \in \mathbb{R}^{m}:|y|<R\right\}, R>0$, induces the composition operator (Nemytskii)

$$
\tilde{f}(u):=f\left(x, u(x), D u(x), \ldots, D^{p} u(x)\right),
$$

where $D^{k} u(x)$ denote the derivatives $\partial_{x}^{\alpha}$ of order $|\alpha|=k$ (the number $m$ of $y$-variables depends on $p, d$ ).

Lemma 2.12. (Lipschitz estimates). Fix $\gamma>0$ and assume that $f \in C^{\infty}\left(\mathbb{T}^{d} \times B_{R} ; \mathbb{R}\right)$. Then, for any $u \in H^{\gamma+p}$ with $\|u\|_{W^{p, \infty}}<R$, one has

$$
\begin{aligned}
& \|\tilde{f}(u)\|_{H^{\gamma}} \leq C\|f\|_{C^{\gamma}}\left(1+\|u\|_{H^{\gamma+p}}\right), \\
& \|\tilde{f}(u+h)-\tilde{f}(u)\|_{H^{\gamma}} \leq C\|f\|_{C^{\gamma+1}}\left(\|h\|_{H^{\gamma+p}}+\|h\|_{W^{p, \infty}}\|u\|_{H^{\gamma+p}}\right), \\
& \left\|\tilde{f}(u+h)-\tilde{f}(u)-\left(d_{u} \tilde{f}\right)(u)[h]\right\|_{H^{\gamma}} \leq \\
& \quad C\|f\|_{C^{\gamma+2}}\|h\|_{W^{p, \infty}}\left(\|h\|_{H^{\gamma+p}}+\|h\|_{W^{p, \infty}}\|u\|_{H^{\gamma+p}}\right),
\end{aligned}
$$

for any $h \in H^{\gamma+p}$ with $\|h\|_{W^{p, \infty}}<R / 2$ and where $C>0$ is a constant depending on $\gamma$ and the norm $\|u\|_{W^{p, \infty}}$.

Consider the function $F(u, \nabla u)$ introduced after formula (1.2) and a symbol $f(\xi)$, independent of $x \in$ $\mathbb{T}^{d}$, such that $|f|_{\mathcal{N}_{s}^{m}} \leq C<+\infty$, for some constant $C$. Let us define the symbol

$$
a(x, \xi):=\left(\partial_{z_{j}^{\alpha} z_{k}^{\beta}} F\right)(u, \nabla u) f(\xi), \quad z_{j}^{\alpha}:=\partial_{x_{j}}^{\alpha} u^{\sigma}, z_{k}^{\beta}:=\partial_{x_{k}}^{\beta} u^{\sigma^{\prime}}
$$

for some $j, k=1, \ldots, d, \alpha, \beta \in\{0,1\}$ and $\sigma, \sigma^{\prime} \in\{ \pm\}$ where we used the notation $u^{+}=u$ and $u^{-}=\bar{u}$. We have the following. 
Lemma 2.13. Fix $s_{0}>d / 2$. For $u \in B_{R}\left(H^{s+s_{0}+1}\left(\mathbb{T}^{d} ; \mathbb{C}\right)\right), s \in \mathbb{N}$, we have

$$
|a|_{\mathcal{N}_{s}^{m}} \lesssim C\|u\|_{H^{s+s_{0}+1}}
$$

where $C>0$ is some constant depending on $\|u\|_{H^{s+s_{0}+1}}$ and bounded from above when u goes to zero. Moreover, for any $h \in H^{s+s_{0}+1}$, the map $h \rightarrow\left(\partial_{u} a\right)(u ; x, \xi) h$ is $a \mathbb{C}$-linear map from $H^{s+s_{0}+1}$ to $\mathbb{C}$ and satisfies

$$
\left|\left(\partial_{u} a\right) h\right|_{\mathcal{N}_{s}^{m}} \lesssim C\|h\|_{H^{s+s_{0}+1}}
$$

for some constant $C>0$ as above. The same holds for $\partial_{\bar{u}} a$.

Proof. It follows by Lemma 2.12 applied on the function $\left(\partial_{z_{j}^{\alpha} z_{k}^{\beta}} F\right)(u, \nabla u) f(\xi)$, see 2.79).

\section{PARALINEARIZATION OF NLS}

We now paralinearize the Hamiltonian nonlinearity $P(u)$ in (1.2).

Lemma 3.1. Fix $s_{0}>d / 2$ and $0 \leq \rho<s-s_{0}, s \geq s_{0}$. Consider $u \in H^{s}\left(\mathbb{T}^{d} ; \mathbb{C}\right)$. Then we have that

$$
\begin{aligned}
& P(u)=T_{\partial_{u \bar{u}} F}[u]+T_{\partial_{\bar{u} \bar{u} F}}[\bar{u}] \\
& +\sum_{j=1}^{d}\left(T_{\partial_{\bar{u} u_{x_{j}}} F}\left[u_{x_{j}}\right]+T_{\partial_{\bar{u} \overline{u_{x_{j}}}}}\left[\overline{u_{x_{j}}}\right]\right)-\sum_{j=1}^{d} \partial_{x_{j}}\left(T_{\partial_{u \overline{u_{x_{j}}}}}[u]+T_{\partial_{\bar{u} \overline{u_{x_{j}}}}}[\bar{u}]\right)
\end{aligned}
$$

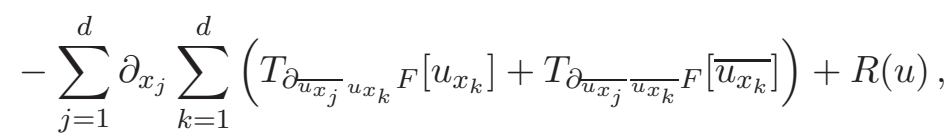

where $R(u)$ is a remainder satisfying

$$
\|R(u)\|_{H^{s+\rho}} \lesssim C\|u\|_{H^{s}}^{2},
$$

for some constant $C>0$ depending on $\|u\|_{H^{s}}$ bounded as $u$ goes to 0 .

Proof. The (3.1)-(3.3) follow by the Bony para-linearization formula, see Lemma 2.7 (see also [27], [32]).

We now rewrite the equation (1.1) as a para-differential system. Let us introduce the symbols

$$
\begin{aligned}
& a_{2}(x, \xi):=a_{2}(U ; x, \xi):=\sum_{j, k=1}^{d}\left(\partial_{\overline{u_{x_{k}}} u_{x_{j}}} F\right) \xi_{j} \xi_{k}, \\
& b_{2}(x, \xi):=b_{2}(U ; x, \xi):=\sum_{j, k=1}^{d}\left(\partial_{\overline{u_{x_{k}}}} \overline{u_{x_{j}}} F\right) \xi_{j} \xi_{k}, \\
& a_{1}(x, \xi):=a_{1}(U ; x, \xi):=\frac{\mathrm{i}}{2} \sum_{j=1}^{d}\left(\left(\partial_{\bar{u} u_{x_{j}}} F\right)-\left(\partial_{u \overline{u_{x_{j}}}} F\right)\right) \xi_{j},
\end{aligned}
$$

where $F=F(u, \nabla u)$ in $(1.3)$.

Lemma 3.2. One has that

$$
\begin{gathered}
a_{2}(x, \xi)=\overline{a_{2}(x, \xi)}, \quad a_{1}(x, \xi)=\overline{a_{1}(x, \xi)}, \quad a_{1}(x,-\xi)=-a_{1}(x, \xi), \quad a_{2}(x,-\xi)=a_{2}(x, \xi), \\
\left|a_{2}\right|_{\mathcal{N}_{p}^{2}}+\left|b_{2}\right|_{\mathcal{N}_{p}^{2}}+\left|a_{1}\right|_{\mathcal{N}_{p}^{1}} \lesssim C\|u\|_{H^{p+s_{0}+1}}, \quad \forall p+s_{0} \leq s, \quad p \in \mathbb{N},
\end{gathered}
$$

for some constant $C>0$ depending on $\|u\|_{H^{p+s_{0}+1}}$ bounded as u goes to 0 .

Proof. The (3.6) follows by direct inspection using (3.5). The (3.7) follows by Lemma 2.13.

The following holds true. 
Proposition 3.3. (Paralinearization of NLS). We have that the equation (1.1) is equivalent to the following system (recall 2.12) ):

$$
\dot{U}=\mathrm{i} E O p^{\mathrm{BW}}\left(|\xi|^{2} \mathbb{1}+A_{2}(x, \xi)+A_{1}(x, \xi)\right) U+R(U) U,
$$

where

$$
U:=\left[\frac{u}{u}\right], \quad E:=\left[\begin{array}{cc}
1 & 0 \\
0 & -1
\end{array}\right], \quad \mathbb{1}:=\left[\begin{array}{ll}
1 & 0 \\
0 & 1
\end{array}\right],
$$

the matrices $A_{2}(x, \xi)=A_{2}(U ; x, \xi), A_{1}(x, \xi)=A_{1}(U ; x, \xi)$ have the form

$$
A_{2}(x, \xi):=\left(\begin{array}{cc}
a_{2}(x, \xi) & b_{2}(x, \xi) \\
b_{2}(x,-\xi) & a_{2}(x, \xi)
\end{array}\right), \quad A_{1}(x, \xi):=\left(\begin{array}{cc}
a_{1}(x, \xi) & 0 \\
0 & \frac{0}{a_{1}(x,-\xi)}
\end{array}\right)
$$

and $a_{2}, a_{1}, b_{2}$ are the symbol in (3.5). The operators $O p^{\mathrm{BW}}\left(A_{i}(x, \xi)\right)$ are self-adjoint (see (2.72)). The remainder $R(U)$ is a $2 \times 2$ matrix of operators (see (2.66) which is real-to-real, i.e. satisfies (2.68). Moreover, for any $s \geq 2(d+1)+3$ and any $U, V \in H^{s}\left(\mathbb{T}^{d} ; \mathbb{C}^{2}\right)$, it satisfies the estimates

$$
\begin{aligned}
\|R(U) U\|_{H^{s}} & \lesssim C\|U\|_{H^{s}}^{2}, \quad C:=C\left(\|U\|_{H^{s}}\right), \\
\|R(U)[U]-R(V)[V]\|_{H^{s}} & \lesssim C_{2}\left(\|U\|_{H^{s}}+\|V\|_{H^{s}}\right)\|U-V\|_{H^{s}}, \quad C_{2}:=C_{2}\left(\|U\|_{H^{s}},\|V\|_{H^{s}}\right),
\end{aligned}
$$

for some constants $C, C_{2}>0$ bounded as $u$ and $v$ go to 0 .

Proof. We start by noting that

$$
\partial_{x_{j}}=O p^{\mathrm{BW}}\left(\mathrm{i} \xi_{j}\right), \quad j=1, \ldots d,
$$

and that the quantization of a symbol $a(x)$ is given by $O p^{\mathrm{BW}}(a(x))$. We also remark that the symbols appearing in (3.1), (3.2) and (3.3) can be estimated (in the norm $\left.|\cdot| \mathcal{N}_{s}^{m}\right)$ by using Lemma 3.2. Consider now the first para-differential term in (3.3). We have, for any $j, k=1, \ldots, d$,

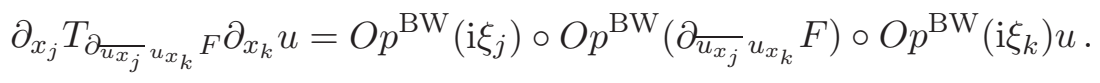

By applying Proposition 2.5 and recalling the Poisson bracket in (2.14), we deduce

$$
\begin{aligned}
O p^{\mathrm{BW}}\left(\mathrm{i} \xi_{j}\right) \circ & O p^{\mathrm{BW}}\left(\partial_{\overline{u_{x_{j}}}} u_{x_{k}} F\right) \circ O p^{\mathrm{BW}}\left(\mathrm{i} \xi_{k}\right)=O p^{\mathrm{BW}}\left(-\xi_{j} \xi_{k} \partial_{\overline{u_{x_{j}}}} u_{x_{k}} F\right) \\
& +O p^{\mathrm{BW}}\left(\frac{\mathrm{i}}{2} \xi_{k} \partial_{x_{j}}\left(\partial_{\overline{u_{x_{j}}}} u_{x_{k}} F\right)-\frac{\mathrm{i} \xi_{j}}{2} \partial_{x_{k}}\left(\partial_{\overline{u_{x_{j}}}} u_{x_{k}} F\right)\right) \\
& +\widetilde{R}_{j, k}^{(1)}(u)+\widetilde{R}_{j, k}^{(2)}(u)
\end{aligned}
$$

where $\widetilde{R}_{j, k}^{(1)}(u):=O p^{\mathrm{BW}}\left(-\frac{1}{4} \partial_{x_{j} x_{k}}\left(\partial_{\overline{u_{x_{j}}} u_{x_{k}}} F\right)\right)$ and $\widetilde{R}_{j, k}^{(2)}(u)$ is some bounded operator. More precisely, using (2.28), (2.16) and the estimates given by Lemma2.13, we have, $\forall h \in H^{s}\left(\mathbb{T}^{d} ; \mathbb{C}\right)$,

$$
\left\|\widetilde{R}_{j, k}^{(2)}(u) h\right\|_{H^{s}} \lesssim C\|h\|_{H^{s}}\|u\|_{H^{s}}, \quad\left\|\widetilde{R}_{j, k}^{(1)}(u) h\right\|_{H^{s}} \lesssim C\|h\|_{H^{s}}\|u\|_{H^{2 s_{0}+3}},
$$

for some constant $C>0$ depending on $\|u\|_{H^{s}}$ bounded as $u$ goes to 0 , with $s_{0} \geq d+1, s_{0} \in \mathbb{N}$. We set

$$
\widetilde{R}(u):=\sum_{j, k=1}^{d}\left(\widetilde{R}_{j, k}^{(1)}(u)+\widetilde{R}_{j, k}^{(2)}(u)\right) .
$$


Then

$$
\begin{aligned}
-\sum_{j, k=1}^{d} \partial_{x_{j}} T_{\partial_{\overline{u_{j}}} u_{x_{k}}} F \partial_{x_{k}} u=O p^{\mathrm{BW}}\left(\sum_{j, k=1}^{d} \xi_{j} \xi_{k} \partial_{\overline{u_{x_{j}}} u_{x_{k}}} F\right)+\widetilde{R}(u) \\
\quad-\frac{\mathrm{i}}{2} O p^{\mathrm{BW}}\left(\sum_{j, k=1}^{d}\left(-\xi_{j} \partial_{x_{k}}\left(\partial_{\overline{u_{x_{j}}} u_{x_{k}}} F\right)+\xi_{k} \partial_{x_{j}}\left(\partial_{\overline{u_{x_{j}}} u_{x_{k}}} F\right)\right)\right) \\
\quad \stackrel{\underline{3.5}}{=} O p^{\mathrm{BW}}\left(a_{2}(x, \xi)\right)+\widetilde{R}(u)+\frac{\mathrm{i}}{2} O p^{\mathrm{BW}}\left(\sum_{j, k=1}^{d} \xi_{j} \partial_{x_{k}}\left(\left(\partial_{\overline{u_{x_{j}}}} u_{x_{k}} F\right)-\left(\partial_{\overline{u_{x_{k}}}} u_{x_{j}} F\right)\right)\right) \\
=O p^{\mathrm{BW}}\left(a_{2}(x, \xi)\right)+\widetilde{R}(u),
\end{aligned}
$$

where we used the symmetry of the matrix $\partial_{\overline{\nabla u} \nabla u} F$ (recall $F$ is real). By performing similar explicit computations on the other summands in (3.1)-(3.3) we get the (3.8), (3.9) with symbols in (3.5). By the discussion above we deduced that the remainder $R(U)$ in (3.8) satisfies the bound (3.10). The estimate (3.11) can be deduced by reasoning as in Lemma 4.5 in [12].

\section{BASIC ENERGY ESTIMATES}

Fix $s_{0} \geq d+1, s_{0} \in \mathbb{N}, s \geq 2 s_{0}+7, T>0$, and consider a function $u$ such that

$$
\begin{aligned}
& u \in L^{\infty}\left([0, T) ; H^{s}\left(\mathbb{T}^{d} ; \mathbb{C}\right)\right) \cap \operatorname{Lip}\left([0, T) ; H^{s-2}\left(\mathbb{T}^{d} ; \mathbb{C}\right)\right), \\
& \sup _{t \in[0, T)}\|u(t)\|_{H^{2 s_{0}+7}}+\sup _{t \in[0, T)}\left\|\partial_{t} u(t)\right\|_{H^{2 s_{0}+5}} \leq \mathrm{r},
\end{aligned}
$$

for some $r>0$. Let $U:=\left[\frac{u}{u}\right] \in \mathcal{U}$ (recall (2.69)). Consider the system

$$
\left\{\begin{array}{l}
\dot{V}=\mathrm{i} E O p^{\mathrm{BW}}\left(|\xi|^{2} \mathbb{1}+A_{2}(x, \xi)+A_{1}(x, \xi)\right) V, \\
V(0)=V_{0}:=U(0),
\end{array}\right.
$$

where $A_{i}, i=1,2$, are the matrices of symbols given by Proposition 3.3 . We shall provide a priori energy estimates for the equation (4.2).

Theorem 4.1. (Energy estimates). Assume (4.1). Then for $s \geq 2 s_{0}+7$ the following holds. If a function $V=\left[\frac{v}{v}\right] \in \mathcal{U}$, with $v \in L^{\infty}\left([0, T) ; H^{s}\left(\mathbb{T}^{d} ; \mathbb{C}\right)\right) \cap \operatorname{Lip}\left([0, T) ; H^{s-2}\left(\mathbb{T}^{d} ; \mathbb{C}\right)\right)$ solves the problem 44.2 , with initial condition $v(0) \in H^{s}\left(\mathbb{T}^{d} ; \mathbb{C}\right)$, then one has

$$
\|v(t)\|_{H^{s}}^{2} \lesssim_{\mathrm{r}}\|v(0)\|_{H^{s}}^{2}+\int_{0}^{t} C\|u(\sigma)\|_{H^{s}}\|v(\sigma)\|_{H^{s}}^{2} d \sigma, \quad \text { for almost every } t \in[0, T),
$$

for some $C>0$ depending on $\|u\|_{H^{s}}$, and bounded from above as $\|u\|_{H^{s}}$ goes to zero.

The proof of the Theorem above requires some preliminary results which will be proved in the following subsections.

4.1. Block-diagonalization. The aim of this section is to block-diagonalize system (4.2) up to bounded remainders. This will be achieved into two steps. In the following, for simplicity, sometimes we omit the dependence on $(x, \xi)$ from the symbols. 
4.1.1. Block-diagonalization at highest order. Consider the matrix of symbols

$$
\begin{aligned}
& E\left(\mathbb{1}+\widetilde{A}_{2, \mathrm{R}}(x, \xi)\right), \quad \widetilde{A}_{2, \mathrm{R}}(x, \xi):=\left(\begin{array}{cc}
\frac{\widetilde{a}_{2, \mathrm{R}}(x, \xi)}{\widetilde{b}_{2, \mathrm{R}}(x,-\xi)} & \widetilde{b}_{2, \mathrm{R}}(x, \xi) \\
\widetilde{a}_{2, \mathrm{R}}(x, \xi)
\end{array}\right), \\
& \widetilde{a}_{2, \mathrm{R}}(x, \xi):=|\xi|^{-2} a_{2}(x, \xi) \mathcal{X}_{\mathrm{R}}(\xi), \quad \widetilde{b}_{2, \mathrm{R}}(x, \xi):=|\xi|^{-2} b_{2}(x, \xi) \mathcal{X}_{\mathrm{R}}(\xi),
\end{aligned}
$$

where $a_{2}(x, \xi)$ and $b_{2}(x, \xi)$ are defined in (3.5) and $\mathcal{X}_{\mathbf{R}}(\xi)$ is the cut-off function in 2.19). Note that the symbols above are well-defined thanks to the cut-off function $\mathcal{X}_{\mathrm{R}}(\xi)$.

Define

$$
\lambda_{2}(x, \xi):=\sqrt{\left(1+\widetilde{a}_{2, \mathrm{R}}(x, \xi)\right)^{2}-\left|\widetilde{b}_{2, \mathrm{R}}(x, \xi)\right|^{2}}, \quad \widetilde{a}_{2, \mathrm{R}}^{+}(x, \xi):=\lambda_{2}(x, \xi)-1 .
$$

Notice that the symbol $\lambda_{2}$ is well-defined by Hypothesis 1.1. The matrix of the normalized eigenvectors associated to the eigenvalues of $E\left(\mathbb{1}+\widetilde{A}_{2, \mathrm{R}}(x, \xi)\right)$ is

$$
\begin{aligned}
S(x, \xi):=\left(\begin{array}{ll}
\frac{s_{1}(x, \xi)}{s_{2}(x, \xi)} & s_{2}(x, \xi) \\
s_{1}(x, \xi)
\end{array}\right), \quad S^{-1}(x, \xi):=\left(\begin{array}{cc}
s_{1}(x, \xi) & -s_{2}(x, \xi) \\
-s_{2}(x, \xi) & s_{1}(x, \xi)
\end{array}\right), \\
s_{1}:=\frac{1+\widetilde{a}_{2, \mathrm{R}}+\lambda_{2}}{\sqrt{2 \lambda_{2}\left(1+\widetilde{a}_{2, \mathrm{R}}+\lambda_{2}\right)}}, \quad s_{2}:=\frac{-\widetilde{b}_{2, \mathrm{R}}}{\sqrt{2 \lambda_{2}\left(1+\widetilde{a}_{2, \mathrm{R}}+\lambda_{2}\right)}} .
\end{aligned}
$$

Let us also define (recall (2.14), (2.15))

$$
\begin{aligned}
S_{1}(x, \xi) & :=\left(\begin{array}{cc}
s_{1}^{(1)}(x, \xi) & s_{2}^{(1)}(x, \xi) \\
s_{2}^{(1)}(x,-\xi) & s_{1}^{(1)}(x,-\xi)
\end{array}\right) \\
& :=\frac{1}{2 \mathrm{i}}\left(\begin{array}{cc}
\left\{s_{2}, \overline{s_{2}}\right\}(x, \xi) & 2\left\{s_{1}, s_{2}\right\}(x, \xi) \\
-2\left\{s_{1}, \overline{s_{2}}\right\}(x,-\xi) & \left\{s_{2}, \overline{s_{2}}\right\}(x,-\xi)
\end{array}\right) S(x, \xi),
\end{aligned}
$$

and

$$
\begin{aligned}
& g_{1}(x, \xi):=\frac{1}{2 \mathrm{i}}\left\{s_{1}^{(1)}, s_{1}\right\}-\frac{1}{2 \mathrm{i}}\left\{s_{2}^{(1)}, \overline{s_{2}}\right\}+\frac{1}{8} \sigma\left(s_{2}, \overline{s_{2}}\right), \\
& g_{2}(x, \xi):=-\frac{1}{2 \mathrm{i}}\left\{s_{1}^{(1)}, s_{2}\right\}+\frac{1}{2 \mathrm{i}}\left\{s_{2}^{(1)}, s_{1}\right\}, \\
& S_{2}(x, \xi):=\left(\begin{array}{cc}
\frac{s_{1}^{(2)}(x, \xi)}{s_{2}^{(2)}(x,-\xi)} & s_{2}^{(2)}(x, \xi) \\
s_{1}^{(2)}(x,-\xi)
\end{array}\right):=-\left(\begin{array}{cc}
\frac{g_{1}(x, \xi)}{g_{2}(x,-\xi)} & g_{2}(x, \xi) \\
g_{1}(x,-\xi)
\end{array}\right) S(x, \xi) .
\end{aligned}
$$

We have the following lemma.

Lemma 4.2. We have that the symbols $\widetilde{a}_{2, \mathrm{R}}^{+}$in (4.5), $\widetilde{a}_{2, \mathrm{R}}, \widetilde{b}_{2, \mathrm{R}}$ in (4.4), $s_{1}, s_{2}$ in (4.6), $g_{1}, g_{2}$ in (4.8) are even in the variable $\xi \in \mathbb{R}^{d}$, while the symbols in the matrix (4.7) are odd in $\xi \in \mathbb{R}^{d}$. Let $s_{0} \geq d+1, p \in \mathbb{N}$. One has

$$
\begin{aligned}
& \left|\widetilde{a}_{2, \mathrm{R}}^{+}\right|_{\mathcal{N}_{p}^{0}}+\left|\widetilde{a}_{2, \mathrm{R}}\right|_{\mathcal{N}_{p}^{0}}+\left|\widetilde{b}_{2, \mathrm{R}}\right|_{\mathcal{N}_{p}^{0}}+\left|s_{1}-1\right|_{\mathcal{N}_{p}^{0}}+\left|s_{2}\right|_{\mathcal{N}_{p}^{0}} \lesssim C_{1}\|u\|_{H^{p+s_{0}+1}}, \quad p+s_{0}+1 \leq s, \\
& \left|\left\{s_{2}, s_{1}\right\}\right|_{\mathcal{N}_{p}^{-1}}+\left|\left\{s_{2}, \overline{s_{2}}\right\}\right|_{\mathcal{N}_{p}^{-1}}+\left|g_{i}\right|_{\mathcal{N}_{p}^{-2}} \lesssim C_{2}\|u\|_{H^{p+s_{0}+3}}, \quad p+s_{0}+3 \leq s,
\end{aligned}
$$

for $i=1,2$, and for some $C_{1}$ depending on $\|u\|_{H^{p+s_{0}+1}}$ and $C_{2}$ depending on $\|u\|_{H^{p+s_{0}+3}}$, both bounded as u goes to zero.

Proof. The symbols are even in $\xi$ by direct inspection using (4.4), (4.6) and (3.6). The symbols in (4.7) are odd in $\xi$ by the same reasoning. Estimates (4.9), 4.10) follow by Lemma 3.2 since the symbols $s_{1}, s_{2}$ are regular functions of $\widetilde{a}_{2, \mathrm{R}}, \widetilde{b}_{2, \mathrm{R}}$ (recall also the $\underline{2.13}$ ). 
By a direct computation one can check that

$$
S^{-1}(x, \xi) E\left(\mathbb{1}+\widetilde{A}_{2, \mathrm{R}}(x, \xi)\right) S(x, \xi)=\left[\begin{array}{cc}
\lambda_{2}(x, \xi) & 0 \\
0 & -\lambda_{2}(x, \xi)
\end{array}\right], \quad s_{1}^{2}-\left|s_{2}\right|^{2}=1 .
$$

Moreover the matrices of symbols $S, S^{-1}$ in (4.6), $S_{1}$ in (4.7) and $S_{2}$ in (4.8) have the form (2.73), i.e. they are real-to-real. We shall study how the system (3.8) transforms under the maps

$$
\begin{aligned}
& \Phi=\Phi(u)[\cdot]:=O p^{\mathrm{BW}}\left(S^{-1}(x, \xi)\right), \\
& \Psi=\Psi(u)[\cdot]:=O p^{\mathrm{BW}}\left(S(x, \xi)+S_{1}(x, \xi)+S_{2}(x, \xi)\right) .
\end{aligned}
$$

Lemma 4.3. Assume the (4.1). For any $s \in \mathbb{R}$ the following holds:

(i) there exists a constant $C$ depending on $s$ and on $\|u\|_{H^{2 s_{0}+3}}$, bounded as $u$ goes to zero, such that

$$
\|\Phi(u) V\|_{H^{s}}+\|\Psi(u) V\|_{H^{s}} \leq\|V\|_{H^{s}}\left(1+C\|u\|_{H^{2 s_{0}+3}}\right), \quad \forall V \in H^{s}\left(\mathbb{T}^{d} ; \mathbb{C}\right) ;
$$

(ii) one has $\Psi(u)[\Phi(u)[\cdot]]=\mathbb{1}+Q(u)[\cdot]$ where $Q$ is a real-to-real remainder of the form (2.66) satisfying

$$
\begin{gathered}
\|Q(u) V\|_{H^{s+3}} \lesssim C\|V\|_{H^{s}}\|u\|_{H^{2 s_{0}+7}}, \\
\|Q(u) V\|_{H^{s+2}} \lesssim C \mathrm{R}^{-1}\|V\|_{H^{s}}\|u\|_{H^{2 s_{0}+7}},
\end{gathered}
$$

for some $C>0$ depending on $\|u\|_{H^{2 s_{0}+7}}$ and bounded as $u$ goes to zero;

(iii) for $\mathrm{R}>0$ large enough with respect to $\mathrm{r}>0$ in 4.1) the map $\mathbb{1}+Q(u)$ is invertible and $(\mathbb{1}+$ $Q(u))^{-1}=\mathbb{1}+\widetilde{Q}(u)$ with

$$
\|\widetilde{Q}(u) V\|_{H^{s+2}} \lesssim C \mathrm{R}^{-1}\|V\|_{H^{s}}\|u\|_{H^{2 s_{0}+7}},
$$

for some $C>0$ as in item $($ ii $)$. Moreover $\Phi^{-1}(u):=(\mathbb{1}+\widetilde{Q}(u)) \Psi(u)$ satisfies

$$
\left\|\Phi^{-1}(u) V\right\|_{H^{s}} \leq\|V\|_{H^{s}}\left(1+C\|u\|_{H^{2 s_{0}+7}}\right), \quad \forall V \in H^{s}\left(\mathbb{T}^{d} ; \mathbb{C}\right),
$$

for some $C>0$ depending on $\|u\|_{H^{2 s_{0}+7}}$ and bounded as $u$ goes to zero;

(iv) for almost any $t \in[0, T)$, one has $\partial_{t} \Phi(u)[\cdot]=O p^{\mathrm{BW}}\left(\partial_{t} S^{-1}(x, \xi)\right)$ and

$$
\left|\partial_{t} S^{-1}(x, \xi)\right|_{\mathcal{N}_{s_{0}}^{0}} \lesssim_{\mathrm{r}} C\|u\|_{H^{2 s_{0}+3}}, \quad\left\|\partial_{t} \Phi(u) V\right\|_{H^{s}} \lesssim_{\mathrm{r}} C\|V\|_{H^{s}}\|u\|_{H^{2 s_{0}+3}},
$$

for some $C>0$ depending on $\|u\|_{H^{2 s_{0}+3}}$ bounded as u goes to zero.

Proof. (i) The bound (4.13) follows by (2.16) and (4.9), (4.10).

(ii) By applying Proposition 2.5 to the maps in (4.12), using the expansion (2.27) and the (4.7), (4.8) we have $\Psi(u)[\Phi(u)[\cdot]]=\mathbb{1}+Q(u)$. The remainder $Q(u)$ satisfies (4.14), (4.15) by estimates (2.28), (2.31) and (4.9), 4.10).

(iii) This item follows by using Neumann series, the second condition in (4.1), the bound (4.15) and taking $\mathrm{R}$ large enough to obtain the smallness condition $\|Q(u) V\|_{H^{s+2}} \lesssim 1 / 2\|V\|_{H^{s}}$. The 44.17 follows by composition using (4.16) and 4.13).

(iv) We note that

$$
\partial_{t} s_{1}(x, \xi)=\left(\partial_{u} s_{1}\right)(u ; x, \xi)[\dot{u}]+\left(\partial_{\bar{u}} s_{1}\right)(u ; x, \xi)[\dot{\bar{u}}] .
$$

By hypothesis (4.1) we have that $\dot{u}$ and $\dot{\bar{u}}$ belong to $H^{s-2}\left(\mathbb{T}^{d} ; \mathbb{C}\right)$. Moreover, recalling (4.6) and (4.4), we can express $\partial_{t} s_{1}(x, \xi)$ in terms of the derivatives of the symbols $a_{2}(x, \xi), b_{2}(x, \xi)$ in (3.5). Therefore, by applying Lemma2.13 (see estimate (2.81), we deduce

$$
\left|\partial_{t} s_{1}(x, \xi)\right|_{\mathcal{N}_{s_{0}}^{0}} \lesssim_{\Upsilon} C\|u\|_{H^{2 s_{0}+3}} .
$$

Reasoning similarly one can prove a similar bound for the symbol $s_{2}$. This implies the first in (4.18). The second one follows by (2.16).

We are ready to prove the following conjugation result. 
Proposition 4.4. (Block-diagonalization). Assume (4.1), consider the system (4.2) and set

$$
Z:=\Phi(u)[V]
$$

Then we have

$$
\dot{Z}=\mathrm{i} E O p^{\mathrm{BW}}\left(|\xi|^{2} \mathbb{1}+A_{2}^{(1)}(x, \xi)+A_{1}^{(1)}(x, \xi)\right) Z+\mathcal{R}(U) V
$$

where (recall 4.5)

$$
\begin{aligned}
& A_{2}^{(1)}(x, \xi):=\left(\begin{array}{cc}
a_{2}^{(1)}(x, \xi) & 0 \\
0 & a_{2}^{(1)}(x, \xi)
\end{array}\right), \quad a_{2}^{(1)}(x, \xi):=|\xi|^{2} \widetilde{a}_{2, \mathrm{R}}^{+}(x, \xi), \\
& A_{1}^{(1)}(x, \xi):=\left(\begin{array}{cc}
a_{1}^{(1)}(x, \xi) & b_{1}^{(1)}(x, \xi) \\
b_{1}^{(1)}(x,-\xi) & a_{1}^{(1)}(x,-\xi)
\end{array}\right), \quad a_{i}^{(1)}(x, \xi) \in \mathbb{R}, i=1,2, \\
& a_{1}^{(1)}(x,-\xi)=-a_{1}^{(1)}(x, \xi), \quad b_{1}^{(1)}(x,-\xi)=-b_{1}^{(1)}(x, \xi) \quad a_{2}^{(1)}(x,-\xi)=a_{2}^{(1)}(x, \xi),
\end{aligned}
$$

and the symbols $a_{2}^{(1)}, a_{1}^{(1)}, b_{1}^{(1)}$ satisfy

$$
\begin{aligned}
&\left|a_{2}^{(1)}\right|_{\mathcal{N}_{p}^{2}} \lesssim c_{1}\|u\|_{H^{p+s_{0}+1}}, \quad p+s_{0}+1 \leq s, \quad p \in \mathbb{N} \\
&\left|a_{1}^{(1)}\right|_{\mathcal{N}_{p}^{1}}+\left|b_{1}^{(1)}\right|_{\mathcal{N}_{p}^{1}} \lesssim c_{2}\|u\|_{H^{p+s_{0}+3}}, \quad p+s_{0}+3 \leq s, \quad p \in \mathbb{N}
\end{aligned}
$$

for some $\mathrm{c}_{1}, \mathrm{c}_{2}>0$ depending respectively on $\|u\|_{H^{p+s_{0}+1}}$ and $\|u\|_{H^{p+s_{0}+3}}$, bounded as $u$ goes to zero. The remainder $\mathcal{R}$ is real-to-real and satisfies, for any $s \geq 2 s_{0}+7$, the estimate

$$
\|\mathcal{R}(U) V\|_{H^{s}} \lesssim_{\mathrm{r}} C\|V\|_{H^{s}}\|u\|_{H^{s}},
$$

for some $C>0$ depending on $\|u\|_{H^{s}}$, bounded as u goes to zero.

Proof. By 4.2), 4.19] we have

$$
\dot{Z}=\Phi(u) \mathrm{i} E O p^{\mathrm{BW}}\left(|\xi|^{2} \mathbb{1}+A_{2}(x, \xi)+A_{1}(x, \xi)\right) V+\left(\partial_{t} \Phi(u)\right) V .
$$

By item (iii) of Lemma 4.3 we can write $V=\Phi^{-1}(u) Z=(\mathbb{1}+\widetilde{Q}(u)) \Psi(u) Z$ with $\widetilde{Q}(u)$ satisfying (4.16). Using this in (4.25) (recall (2.19) ) we get

$$
\dot{Z}=\Phi(u) \mathrm{i} E O p^{\mathrm{BW}}\left(|\xi|^{2} \mathbb{1}+\mathcal{X}_{\mathrm{R}}(\xi)\left(A_{2}(x, \xi)+A_{1}(x, \xi)\right)\right) \Psi(u) Z+Q_{1}(u) V,
$$

where

$$
\begin{aligned}
Q_{1}(u) & :=\Phi(u) \mathrm{i} E O p^{\mathrm{BW}}\left(\left(1-\mathcal{X}_{\mathrm{R}}(\xi)\right)\left(A_{2}(x, \xi)+A_{1}(x, \xi)\right)\right) \Psi(u) \Phi(u)+\left(\partial_{t} \Phi(u)\right) \\
& +\Phi(u) \mathrm{i} E O p^{\mathrm{BW}}\left(|\xi|^{2} \mathbb{1}+A_{2}(x, \xi)+A_{1}(x, \xi)\right) \widetilde{Q}(u) \Psi(u) \Phi(u) .
\end{aligned}
$$

By using (4.13), (4.14), 4.16), (2.16), (2.20), the (2.80) on the symbols $a_{2}, b_{2}, a_{1}$, and item (iv) of Lemma 4.3 we deduce

$$
\left\|Q_{1}(u) V\right\|_{H^{s}} \lesssim_{\mathrm{r}} C \mathrm{R}^{2}\|V\|_{H^{s}}\|u\|_{H^{s}}, \quad s \geq 2 s_{0}+7,
$$

for some constant $C>0$ depending on $\|u\|_{H^{s}}$, bounded as $u$ goes to zero. We now study the term of order one in (4.26). Recalling (4.12) we have

$$
\Phi(u) \mathrm{i} E O p^{\mathrm{BW}}\left(\mathcal{X}_{\mathrm{R}} A_{1}(x, \xi)\right) \Psi(u)=O p^{\mathrm{BW}}\left(S^{-1}\right) \mathrm{i} E O p^{\mathrm{BW}}\left(\mathcal{X}_{\mathrm{R}} A_{1}\right) O p^{\mathrm{BW}}(S)+Q_{2}(u),
$$

where

$$
Q_{2}(u):=\Phi(u) \mathrm{i} E O p^{\mathrm{BW}}\left(\mathcal{X}_{\mathrm{R}} A_{1}\right) O p^{\mathrm{BW}}\left(S_{1}+S_{2}\right) .
$$

Using Lemmata 3.2, 4.2, 2.3 (recall that $S_{1}, S_{2}$ in 4.7), (4.8) are matrices of symbols of order $\leq-1$ ) one gets

$$
\left\|Q_{2}(u) V\right\|_{H^{s}} \lesssim C\|V\|_{H^{s}}\|u\|_{H^{2 s_{0}+3}}
$$


for some constant $C>0$ depending on $\|u\|_{H^{2 s_{0}+3}}$, bounded as $u$ goes to zero. We define

$$
a_{i, \mathrm{R}}(x, \xi):=\mathcal{X}_{\mathrm{R}}(\xi) a_{i}(x, \xi), \quad i=1,2,
$$

with $a_{2}(x, \xi), a_{1}(x, \xi)$ in (3.5). Recalling Lemma 3.2 (2.12) and (4.6) we have

$$
\begin{aligned}
& O p^{\mathrm{BW}}\left(S^{-1}\right) O p^{\mathrm{BW}}\left(\mathrm{i} E \mathcal{X}_{\mathrm{R}} A_{1}\right) O p^{\mathrm{BW}}(S)=\mathrm{i} E\left(\frac{C_{1}}{C_{2}} \frac{C_{2}}{C_{1}}\right) \\
& C_{1}:=T_{s_{1}} T_{a_{1, \mathrm{R}}} T_{s_{1}}-T_{s_{2}} T_{a_{1, \mathrm{R}}} T_{\overline{s_{2}}}, \quad C_{2}:=T_{s_{1}} T_{a_{1, \mathrm{R}}} T_{s_{2}}-T_{s_{2}} T_{a_{1, \mathrm{R}}} T_{s_{1}} .
\end{aligned}
$$

By using Proposition 2.5 and the second condition in (4.11) we get (see the expansion 2.27)

$$
C_{1}=T_{a_{1, \mathrm{R}}}+Q_{3}(u), \quad C_{2}=Q_{4}(u)
$$

where $Q_{i}(u), i=3,4$ are remainders satisfying, using Lemmata 3.2, 4.2 ,

$$
\left\|Q_{i}(u) V\right\|_{H^{s}} \lesssim C\|V\|_{H^{s}}\|u\|_{H^{2 s_{0}+5}}
$$

for some constant $C>0$ depending on $\|u\|_{H^{2 s_{0}+5}}$, bounded as $u$ goes to zero. Let us study the term of order two in 4.26). By an explicit computation, using Proposition 2.5 and Lemma 4.2, we have

$$
\begin{aligned}
& \Phi(u) \mathrm{i} E O p^{\mathrm{BW}}\left(\left(|\xi|^{2} \mathbb{1}+\mathcal{X}_{\mathrm{R}}(\xi)\right.\right.\left.\left.A_{2}(x, \xi)\right)\right) \Psi(u)=\mathrm{i} E\left(\frac{B_{1}}{B_{2}} \frac{B_{2}}{B_{1}}\right) \\
&\left.+O p^{\mathrm{BW}}\left(S^{-1} \mathrm{i} E\left(|\xi|^{2} \mathbb{1}+\mathcal{X}_{\mathrm{R}}(\xi) A_{2}\right) S_{1}\right)\right)+Q_{5}(u)
\end{aligned}
$$

where

$$
\left\|Q_{5}(u) V\right\|_{H^{s}} \lesssim C\|V\|_{H^{s}}\|u\|_{H^{2 s_{0}+7}},
$$

for some $C>0$ depending on $\|u\|_{H^{2 s_{0}+7}}$, bounded as $u$ goes to zero, and where, recalling Lemma 3.2 , (2.12) and (4.6), 4.31,

$$
\begin{aligned}
& B_{1}:=T_{s_{1}} T_{|\xi|^{2}+a_{2, \mathrm{R}}} T_{s_{1}}+T_{s_{1}} T_{b_{2, \mathrm{R}}} T_{\overline{s_{2}}}+T_{s_{2}} T_{\overline{b_{2, \mathrm{R}}}} T_{s_{1}}+T_{s_{2}} T_{|\xi|^{2}+a_{2, \mathrm{R}}} T_{\overline{s_{2}}}, \\
& B_{2}:=T_{s_{1}} T_{|\xi|^{2}+a_{2, \mathrm{R}}} T_{s_{2}}+T_{s_{1}} T_{b_{2, \mathrm{R}}} T_{s_{1}}+T_{s_{2}} T_{\overline{b_{2, \mathrm{R}}}} T_{s_{2}}+T_{s_{2, \mathrm{R}}} T_{|\xi|^{2}+a_{2, \mathrm{R}}} T_{s_{1}} .
\end{aligned}
$$

We study each term separately. By using Proposition 2.5 we get (see the expansion 2.27)

$$
B_{1}:=T_{c_{2}}+T_{c_{1}}+Q_{6}(u)
$$

where

$$
\left\|Q_{6}(u) h\right\|_{H^{s}} \lesssim C\|h\|_{H^{s}}\|u\|_{H^{2 s_{0}+5}},
$$

for some constant $C>0$ depending on $\|u\|_{H^{2 s_{0}+5}}$, bounded as $u$ goes to zero, and

$$
\begin{aligned}
c_{2}(x, \xi) & :=\left(|\xi|^{2}+a_{2, \mathrm{R}}\right)\left(s_{1}^{2}+\left|s_{2}\right|^{2}\right)+b_{2, \mathrm{R}} s_{1} \overline{s_{2}}+\overline{b_{2, \mathrm{R}}} s_{1} s_{2}, \\
c_{1}(x, \xi) & :=\frac{1}{2 \mathrm{i}}\left\{s_{1},\left(|\xi|^{2}+a_{2, \mathrm{R}}\right) s_{1}\right\}+\frac{s_{1}}{2 \mathrm{i}}\left\{|\xi|^{2}+a_{2, \mathrm{R}}, s_{1}\right\} \\
& +\frac{1}{2 \mathrm{i}}\left\{s_{1}, b_{2, \mathrm{R}} \overline{s_{2}}\right\}+\frac{s_{1}}{2 \mathrm{i}}\left\{b_{2, \mathrm{R}}, \overline{s_{2}}\right\}+\frac{1}{2 \mathrm{i}}\left\{s_{2}, \overline{b_{2, \mathrm{R}}} s_{1}\right\} \\
& +\frac{s_{2}}{2 \mathrm{i}}\left\{\overline{b_{2, \mathrm{R}}}, s_{1}\right\}+\frac{1}{2 \mathrm{i}}\left\{s_{2},\left(|\xi|^{2}+a_{2, \mathrm{R}}\right) \overline{s_{2}}\right\}+\frac{s_{2}}{2 \mathrm{i}}\left\{|\xi|^{2}+a_{2, \mathrm{R}}, \overline{s_{2}}\right\} .
\end{aligned}
$$

By expanding the Poisson bracket (see (2.14) we get that

$$
c_{2}(x, \xi)=\overline{c_{2}(x, \xi)}, \quad c_{1}(x, \xi)=\overline{c_{1}(x, \xi)}, \quad c_{1}(x,-\xi)=-c_{1}(x, \xi) .
$$

Moreover, by (4.9), 2.13) and Lemma2.13 on $a_{2}, b_{2}$, we have

$$
\left|c_{1}\right|_{\mathcal{N}_{p}^{1}} \lesssim C\|u\|_{H^{p+s_{0}+2}},
$$

for some $C$ depending on $\|u\|_{H^{p+s_{0}+2}}$, bounded as $u$ goes to zero. Reasoning similarly we deduce (see (4.36)

$$
B_{2}:=T_{d_{2}}+T_{d_{1}}+Q_{7}(u)
$$


where

$$
\left\|Q_{7}(u) h\right\|_{H^{s}} \lesssim C\|h\|_{H^{s}}\|u\|_{H^{2 s_{0}+5}},
$$

for some $C$ depending on $\|u\|_{H^{2 s_{0}+5}}$, bounded as $u$ goes to zero, and

$$
\begin{aligned}
d_{2}(x, \xi) & :=\left(|\xi|^{2}+a_{2, \mathrm{R}}\right) s_{1} s_{2}+b_{2} s_{1}^{2}+\overline{b_{2, \mathrm{R}}} s_{2}^{2}, \\
d_{1}(x, \xi) & :=\frac{1}{2 \mathrm{i}}\left\{s_{1},\left(|\xi|^{2}+a_{2, \mathrm{R}}\right) s_{2}\right\}+\frac{s_{1}}{2 \mathrm{i}}\left\{|\xi|^{2}+a_{2, \mathrm{R}}, s_{2}\right\} \\
& +\frac{1}{2 \mathrm{i}}\left\{s_{1}, b_{2, \mathrm{R}} s_{1}\right\}+\frac{s_{1}}{2 \mathrm{i}}\left\{b_{2, \mathrm{R}}, s_{1}\right\}+\frac{1}{2 \mathrm{i}}\left\{s_{2}, \overline{b_{2, \mathrm{R}}} s_{2}\right\} \\
& +\frac{s_{2}}{2 \mathrm{i}}\left\{\overline{b_{2, \mathrm{R}}}, s_{2}\right\}+\frac{1}{2 \mathrm{i}}\left\{s_{2},\left(|\xi|^{2}+a_{2, \mathrm{R}}\right) s_{1}\right\}+\frac{s_{2}}{2 \mathrm{i}}\left\{|\xi|^{2}+a_{2, \mathrm{R}}, s_{1}\right\} .
\end{aligned}
$$

By expanding the Poisson bracket (see (2.14) we get that

$$
d_{1}(x, \xi) \equiv 0 .
$$

We now study the second summand in the right hand side of (4.34 by computing explicitly the matrix of symbols of order 1. Using (4.6), (4.7), 4.11), we get

$$
S^{-1} E\left(|\xi|^{2} \mathbb{1}+\mathcal{X}_{\mathrm{R}}(\xi) A_{2}(x, \xi)\right) S_{1}=E\left(\frac{r_{1}(x, \xi)}{r_{2}(x,-\xi)} \frac{r_{2}(x, \xi)}{r_{1}(x,-\xi)}\right)
$$

where

$$
\begin{aligned}
& r_{1}(x, \xi):=\left(|\xi|^{2}+a_{2, \mathrm{R}}\right) s_{1} s_{1}^{(1)}+b_{2, \mathrm{R}} s_{1} \overline{s_{2}^{(1)}}+\overline{b_{2, \mathrm{R}}} s_{2} s_{1}^{(1)}+\left(|\xi|^{2}+a_{2, \mathrm{R}}\right) s_{2}{s_{2}^{(1)}}^{(1)} \\
& r_{2}(x, \xi):=\left(|\xi|^{2}+a_{2, \mathrm{R}}\right) s_{1} s_{2}^{(1)}+b_{2, \mathrm{R}} s_{1} s_{1}^{(1)}+\overline{b_{2, \mathrm{R}}} s_{2} s_{2}^{(1)}+\left(|\xi|^{2}+a_{2, \mathrm{R}}\right) s_{2} s_{1}^{(1)} .
\end{aligned}
$$

Moreover, using Lemma 4.2, one can check that the symbols $r_{1}, r_{2}$ satisfy

$$
r_{1}(x, \xi)=\overline{r_{1}(x, \xi)}, \quad r_{1}(x,-\xi)=-r_{1}(x, \xi), \quad r_{2}(x,-\xi)=-r_{2}(x, \xi),
$$

and, using (4.9) and 4.10), we can note

$$
\left|r_{i}\right|_{\mathcal{N}_{p}^{1}} \lesssim C\|u\|_{H^{p+s_{0}+3}}, \quad p+s_{0}+3 \leq s, \quad i=1,2
$$

for some $C>0$ depending on $\|u\|_{H^{p+s_{0}+3}}$, bounded as $u$ goes to zero. By the discussion above we deduce that

$$
\text { (4.29) + (4.34) }=\mathrm{i} E O p^{\mathrm{BW}}\left(\begin{array}{cc}
\frac{c_{2}(x, \xi)}{d_{2}(x,-\xi)} & d_{2}(x, \xi) \\
c_{2}(x, \xi)
\end{array}\right)+\mathrm{i} E O p^{\mathrm{BW}}\left(\begin{array}{cc}
\frac{a_{1}^{(1)}(x, \xi)}{b_{1}^{(1)}(x,-\xi)} & b_{1}^{(1)}(x, \xi) \\
a_{1}^{(1)}(x,-\xi)
\end{array}\right)
$$

where

$$
a_{1}^{(1)}(x, \xi):=a_{1}(x, \xi)+c_{1}(x, \xi)+r_{1}(x, \xi), \quad b_{1}^{(1)}(x, \xi):=r_{2}(x, \xi) .
$$

up to a remainder satisfying (4.24) (see (4.28), (4.30), (4.33), (4.35), (4.38), (4.43)). The symbols $a_{1}^{(1)}(s, \xi)$ and $b_{1}^{(1)}(s, \xi)$ satisfy the parity conditions (4.21) by (4.40), (4.48), and the estimates (4.23) by Lemma 3.2, and estimates 4.41) and 4.49). By (4.39), 4.44) we observe that

$$
\left(\begin{array}{cc}
c_{2}(x, \xi) & d_{2}(x, \xi) \\
-\overline{d_{2}(x,-\xi)} & -c_{2}(x, \xi)
\end{array}\right)=S^{-1}(x, \xi) E\left(\mathbb{1}+\widetilde{A}_{2, \mathrm{R}}(x, \xi)\right) S(x, \xi)|\xi|^{2 \stackrel{4.111}{=}}\left[\begin{array}{cc}
\lambda_{2}(x, \xi) & 0 \\
0 & -\lambda_{2}(x, \xi)
\end{array}\right]|\xi|^{2} .
$$

Therefore the (4.50), (4.52) implies the 4.20). This concludes the proof. 
4.1.2. Block-diagonalization at order 1 . In this section we eliminate the off-diagonal symbol $b_{1}^{(1)}(x, \xi)$ appearing in 4.20), 4.21). In order to do this we consider the symbol

$$
c(x, \xi):=\frac{\mathcal{X}_{\mathrm{R}}(\xi) b_{1}^{(1)}(x, \xi)}{2\left(|\xi|^{2}+a_{2}^{(1)}(x, \xi)\right)}, \quad B(x, \xi):=\left(\begin{array}{cc}
0 & c(x, \xi) \\
c(x,-\xi) & 0
\end{array}\right),
$$

where $a_{2}^{(1)}(x, \xi), b_{1}^{(1)}(x, \xi)$ are given by Proposition 4.4 and $\mathcal{X}_{\mathrm{R}}(\xi)$ is in 2.19). We set

$$
\Phi_{2}(u)[\cdot]:=\mathbb{1}+O p^{\mathrm{BW}}(B(x, \xi)), \quad \Psi_{2}(u)[\cdot]:=\mathbb{1}-O p^{\mathrm{BW}}\left(B(x, \xi)-B^{2}(x, \xi)\right),
$$

where $\mathbb{1}:=\left[\begin{array}{ll}1 & 0 \\ 0 & 1\end{array}\right]$ is the identity matrix. We have the following.

Lemma 4.5. Assume the (4.1). For any $s \in \mathbb{R}$ the following holds:

(i) there exists a constant $C$ depending on $\|u\|_{H^{2 s_{0}+3}}$, bounded as $u$ goes to zero, such that

$$
\left\|\Phi_{2}(u) V\right\|_{H^{s}}+\left\|\Psi_{2}(u) V\right\|_{H^{s}} \leq\|V\|_{H^{s}}\left(1+C\|u\|_{H^{2 s_{0}+3}}\right), \quad \forall V \in H^{s} ;
$$

(ii) one has $\Psi_{2}(u)\left[\Phi_{2}(u)[\cdot]\right]=\mathbb{1}+R_{2}(u)[\cdot]$ where $R_{2}$ is a real-to-real remainder satisfying

$$
\begin{gathered}
\left\|R_{2}(u) V\right\|_{H^{s+3}} \lesssim C\|V\|_{H^{s}}\|u\|_{H^{2 s_{0}+7}}, \\
\left\|R_{2}(u) V\right\|_{H^{s+2}} \lesssim C \mathrm{R}^{-1}\|V\|_{H^{s}}\|u\|_{H^{2 s_{0}+7}},
\end{gathered}
$$

for some $C>0$ depending on $\|u\|_{H^{2 s_{0}+7}}$, bounded as u goes to zero;

(iii) for $\mathrm{R}>0$ large enough with respect to $\mathrm{r}>0$ in 4.1) the map $\mathbb{1}+R_{2}(u)$ is invertible and $(\mathbb{1}+$ $\left.R_{2}(u)\right)^{-1}=\mathbb{1}+\widetilde{R}_{2}(u)$ with

$$
\left\|\widetilde{R}_{2}(u) V\right\|_{H^{s+2}} \lesssim C \mathrm{R}^{-1}\|V\|_{H^{s}}\|u\|_{H^{2 s_{0}+7}}
$$

for some $C>0$ depending on $\|u\|_{H^{2 s_{0}+7}}$, bounded as $u$ goes to zero. Moreover $\Phi_{2}^{-1}(u):=$ $\left(\mathbb{1}+\widetilde{R}_{2}(u)\right) \Psi_{2}(u)$ satisfies

$$
\left\|\Phi_{2}^{-1}(u) V\right\|_{H^{s}} \leq\|V\|_{H^{s}}\left(1+C\|u\|_{H^{2 s_{0}+7}}\right), \quad \forall V \in H^{s}\left(\mathbb{T}^{d} ; \mathbb{C}\right),
$$

for some $C>0$ depending on $\|u\|_{H^{2 s_{0}+7}}$, bounded as u goes to zero;

(iv) for almost any $t \in[0, T)$, one has $\partial_{t} \Phi_{2}(u)[\cdot]=O p^{\mathrm{BW}}\left(\partial_{t} B(x, \xi)\right)$ and

$$
\left|\partial_{t} B(x, \xi)\right|_{\mathcal{N}_{s_{0}}^{-1}} \lesssim_{r} C\|u\|_{H^{2 s_{0}+5}}, \quad\left\|\partial_{t} \Phi_{2}(u) V\right\|_{H^{s+1}} \lesssim_{r} C\|V\|_{H^{s}}\|u\|_{H^{2 s_{0}+5}},
$$

for some $C>0$ depending on $\|u\|_{H^{2 s_{0}+5}}$, bounded as u goes to zero.

Proof. (i) By (4.22), (4.23) and (4.53) we deduce that

$$
|c|_{\mathcal{N}_{p}^{-1}} \lesssim_{p} C\|u\|_{H^{p+s_{0}+3}}, \quad p+s_{0}+3 \leq s,
$$

for some $C>0$ depending on $\|u\|_{H^{p+s_{0}+3}}$, bounded as $u$ goes to zero. The bound (4.55) follows by (2.16) and 4.61).

(ii) By applying Lemma 2.3, Proposition 2.5, using (4.54) and (4.61), we obtain the (4.56). The (4.57) follows by item $(i i)$ of Proposition 2.5 .

(iii) This item follows by using Neumann series, the (4.1), bound (4.57) and taking $\mathrm{R}$ large enough to obtain the smallness condition $\left\|R_{2}(u) h\right\|_{H^{s+2}} \lesssim 1 / 2\|V\|_{H^{s}}$.

(iv) This item follows by (4.53), using the explicit formulæ (4.51), 4.47) and reasoning as in the proof of item $(i v)$ of Lemma 4.3 .

We are ready to prove the following conjugation result. 
Proposition 4.6. (Block-diagonalization at order 1). Assume 4.17, consider the system (4.20) and set (see (4.19))

$$
W:=\Phi_{2}(u)[Z]
$$

Then we have

$$
\dot{W}=\mathrm{i} E O p^{\mathrm{BW}}\left(\begin{array}{cc}
|\xi|^{2}+a_{2}^{(1)}(x, \xi)+a_{1}^{(1)}(x, \xi) & 0 \\
0 & |\xi|^{2}+a_{2}^{(1)}(x, \xi)+a_{1}^{(1)}(x,-\xi)
\end{array}\right) W+\mathcal{R}_{2}(U) V
$$

where $a_{2}^{(1)}(x, \xi), a_{1}^{(1)}(x, \xi)$ are given in Proposition 4.4 and the remainder $\mathcal{R}_{2}$ is real to real and satisfies, for any $s \geq 2 s_{0}+7$, the estimate

$$
\left\|\mathcal{R}_{2}(U) V\right\|_{H^{s}} \lesssim_{\mathrm{r}} C\|V\|_{H^{s}}\|u\|_{H^{s}}
$$

for some $C>0$ depending on $\|u\|_{H^{s}}$, bounded as u goes to zero.

Proof. By (4.20) and (4.62) we have

$$
\dot{W}=\Phi_{2}(u) \mathrm{i} E O p^{\mathrm{BW}}\left(|\xi|^{2} \mathbb{1}+A_{2}^{(1)}(x, \xi)+A_{1}^{(1)}(x, \xi)\right) Z+\Phi_{2}(u) \mathcal{R}(U) V+\left(\partial_{t} \Phi_{2}(u)\right) Z .
$$

By item $(i i i)$ of Lemma 4.5 we can write $Z=\Phi_{2}^{-1}(u) W=\left(\mathbb{1}+\widetilde{R}_{2}(u)\right) \Psi_{2}(u) W$ with $\widetilde{R}_{2}(u)$ satisfying 4.58). Then we have

$$
\dot{W}=\Phi_{2}(u) \mathrm{i} E O p^{\mathrm{BW}}\left(|\xi|^{2} \mathbb{1}+A_{2}^{(1)}(x, \xi)+\mathcal{X}_{\mathrm{R}}(\xi) A_{1}^{(1)}(x, \xi)\right) \Psi_{2}(u) W+G(u) V,
$$

where (recall 4.19), 4.62)

$$
\begin{aligned}
G(u) & :=\Phi_{2}(u) \mathrm{i} E O p^{\mathrm{BW}}\left(|\xi|^{2} \mathbb{1}+A_{2}^{(1)}(x, \xi)+A_{1}^{(1)}(x, \xi)\right) \widetilde{R}_{2}(u) \Psi_{2}(u) \Phi_{2}(u) \Phi(u) \\
& +\Phi_{2}(u) \mathrm{i} E O p^{\mathrm{BW}}\left(\left(1-\mathcal{X}_{\mathrm{R}}(\xi)\right) A_{1}^{(1)}(x, \xi)\right) \Psi_{2}(u) \Phi_{2}(u) \Phi(u) \\
& +\Phi_{2}(u) \mathcal{R}(U)+\partial_{t} \Phi_{2}(u) \Phi(u)
\end{aligned}
$$

Using Lemmata 2.3, 4.3, 4.5 one can check that $G(u)$ satisfies the bound (4.64). Reasoning similarly, and recalling (4.54), we have that

$$
\Phi_{2}(u)\left(\mathrm{i} E O p^{\mathrm{BW}}\left(\mathcal{X}_{\mathrm{R}}(\xi) A_{1}^{(1)}(x, \xi)\right)\right) \Psi_{2}(u)=\mathrm{i} E O p^{\mathrm{BW}}\left(\mathcal{X}_{\mathrm{R}}(\xi) A_{1}^{(1)}(x, \xi)\right)+G_{1}(u)
$$

for some $G_{1}(u)$ satisfying (4.64). By Proposition 2.5 we deduce that

$$
\begin{aligned}
\Phi_{2}(u) \mathrm{i} E O p^{\mathrm{BW}} & \left(|\xi|^{2} \mathbb{1}+A_{2}^{(1)}(x, \xi)\right) \Psi_{2}(u)= \\
& =\mathrm{i} E O p^{\mathrm{BW}}\left(|\xi|^{2} \mathbb{1}+A_{2}^{(1)}(x, \xi)+\left(\begin{array}{cc}
0 & d(x, \xi) \\
d(x,-\xi) & 0
\end{array}\right)\right)+G_{2}(u)
\end{aligned}
$$

where

$$
d(x, \xi):=-2 c(x, \xi)\left(|\xi|^{2}+a_{2}^{(1)}(x, \xi)\right),
$$

and where $G_{2}(u)$ is some bounded remainder satisfying (4.64). Using (4.53) we deduce that

$$
d(x, \xi)+\mathcal{X}_{\mathrm{R}}(\xi) b_{1}^{(1)}(x, \xi)=0 .
$$

By the discussion above (recall (4.65) ) we have obtained the 4.63. 
4.2. Proof of Theorem 4.1. In this section we prove the energy estimate (4.3). We first need some preliminary results.

Lemma 4.7. Assume (4.1) and consider the function $W$ in (4.62). For any $s \in \mathbb{R}$ one has that

$$
\|W\|_{H^{s}} \sim_{\mathrm{r}}\|V\|_{H^{s}}
$$

where $\mathrm{r}$ is in 4.1).

Proof. Recalling (4.19), (4.62) we write $W=\Phi_{2}(u) Z=\Phi_{2}(u) \Phi(u) V$. By Lemmata4.3, 4.5 we also have $V=\Phi^{-1}(u) \Phi_{2}^{-1}(u) W$. By estimates (4.13), 4.55), (4.17) and 4.59) we have

$$
\begin{aligned}
& \|W\|_{H^{s}} \leq\|V\|_{H^{s}}\left(1+C\|u\|_{H^{2 s_{0}+7}}\right) \\
& \|V\|_{H^{s}} \leq\|W\|_{H^{s}}\left(1+C\|u\|_{H^{2 s_{0}+7}}\right)
\end{aligned}
$$

for some constant $C$ depending on $\|u\|_{H^{2 s_{0}+7}}$, bounded as $u$ goes to zero.

Our aim is to estimate the norm of $V$ by using that $W$ solves the problem (4.63). Let us define the symbol

$$
\mathcal{L}:=\mathcal{L}(x, \xi):=|\xi|^{2}+a_{2}^{(1)}(x, \xi),
$$

where $a_{2}^{(1)}(x, \xi)$ is given in 4.21). Notice that, by 4.4), 4.5), we deduce

$$
a_{2}^{(1)}(x, \xi) \neq 0 \Rightarrow|\xi| \gtrsim \mathrm{R} .
$$

We now study some properties of the operator $T_{\mathcal{L}}$ defined in 4.69).

Lemma 4.8. Assume the (4.1) and let $\gamma \in \mathbb{R}, \gamma>0$. Then, for $\mathrm{R}>0$ large enough (with respect to $\mathrm{r}>0$ in (4.1)), the following holds true.

(i) The symbols $\mathcal{L},(1+\mathcal{L})^{ \pm \gamma}$ satisfy

$$
|\mathcal{L}|_{\mathcal{N}_{s_{0}}^{2}}+\left|(1+\mathcal{L})^{\gamma}\right|_{\mathcal{N}_{s_{0}}^{2 \gamma}}+\left|(1+\mathcal{L})^{-\gamma}\right|_{\mathcal{N}_{s_{0}}^{-2 \gamma}} \leq 1+C\|u\|_{H^{2 s_{0}+1}}
$$

for some $C>0$ depending on $\|u\|_{H^{2 s_{0}+1}}$, bounded as u goes to zero.

(ii) For any $s \in \mathbb{R}$ and any $h \in H^{s}\left(\mathbb{T}^{d} ; \mathbb{C}\right)$, one has

$$
\begin{gathered}
\left\|T_{\mathcal{L}} h\right\|_{H^{s-2}}+\left\|T_{(1+\mathcal{L})^{\gamma}} h\right\|_{H^{s-2 \gamma}}+\left\|T_{(1+\mathcal{L})^{-\gamma}} h\right\|_{H^{s+2 \gamma}} \leq\|h\|_{H^{s}}\left(1+C\|u\|_{H^{2 s_{0}+1}}\right), \\
\left\|\left[T_{(1+\mathcal{L})^{\gamma}}, T_{\mathcal{L}}\right] h\right\|_{H^{s-2 \gamma}} \lesssim C\|h\|_{H^{s}}\|u\|_{H^{2 s_{0}+5}},
\end{gathered}
$$

for some $C>0$ depending on $\|u\|_{H^{2 s_{0}+5}}$, bounded as u goes to zero.

(iii) One has that $T_{(1+\mathcal{L})^{-\gamma}} T_{(1+\mathcal{L})^{\gamma}}=\mathbb{1}+R(u)[\cdot]$ and, for any $s \in \mathbb{R}$ and any $h \in H^{s}\left(\mathbb{T}^{d} ; \mathbb{C}\right)$,

$$
\begin{aligned}
\|R(u) h\|_{H^{s+2}} & \lesssim C\|h\|_{H^{s}}\|u\|_{H^{2 s_{0}+5}}, \\
\|R(u) h\|_{H^{s+1}} & \lesssim C \mathrm{R}^{-1}\|h\|_{H^{s}}\|u\|_{H^{2 s_{0}+5}},
\end{aligned}
$$

for some $C>0$ depending on $\|u\|_{H^{2 s_{0}+5}}$, bounded as u goes to zero.

(iv) For $\mathrm{R} \gg \mathrm{r}$ sufficiently large, the operator $T_{(1+\mathcal{L})^{\gamma}}$ has a left-inverse $T_{(1+\mathcal{L})^{\gamma}}^{-1}$. For any $s \in \mathbb{R}$ one has

$$
\left\|T_{(1+\mathcal{L})^{\gamma}}^{-1} h\right\|_{H^{s+2 \gamma}} \leq\|h\|_{H^{s}}\left(1+C\|u\|_{H^{2 s_{0}+5}}\right), \quad \forall h \in H^{s}\left(\mathbb{T}^{d} ; \mathbb{C}\right),
$$

for some $C>0$ depending on $\|u\|_{H^{2 s_{0}+5}}$, bounded as u goes to zero.

$(v)$ For almost any $t \in[0, T)$ one have

$$
\left|\partial_{t} a_{2}^{(1)}\right|_{\mathcal{N}_{s_{0}}^{2}} \lesssim_{\mathrm{r}} C\|u\|_{H^{2 s_{0}+3}} .
$$

Moreover

$$
\left\|\left(T_{\partial_{t}(1+\mathcal{L})^{\gamma}}\right) h\right\|_{H^{s-2 \gamma}} \lesssim_{\mathrm{r}} C\|h\|_{H^{s}}\|u\|_{H^{2 s_{0}+3}}, \quad \forall h \in H^{s}\left(\mathbb{T}^{d} ; \mathbb{C}\right)
$$

for some $C>0$ depending on $\|u\|_{H^{2 s_{0}+3}}$, bounded as u goes to zero.

(vi) The operators $T_{\mathcal{L}}, T_{\mathcal{L}^{-1}}$ are self-adjoint with respect to the $L^{2}$-scalar product $(2.5)$. 
Proof. (i) It follows by (4.22) and (4.69).

(ii) The bound (4.72) follows by Lemma 2.3 and 4.71). Let us check the (4.73). By Proposition 2.5 we deduce that (recall formulæ (2.14), 2.15)

$$
\left[T_{(1+\mathcal{L})^{\gamma}}, T_{\mathcal{L}}\right]=O p^{\mathrm{BW}}\left(\frac{1}{\mathrm{i}}\left\{(1+\mathcal{L})^{\gamma}, \mathcal{L}\right\}\right)+R(u)
$$

where the remainder $R(u)$ satisfies (see (2.28) and (4.71))

$$
\|R(u) h\|_{H^{s-2 \gamma+1}} \lesssim C\|h\|_{H^{s}}\|u\|_{2 s_{0}+5},
$$

for some $C>0$ depending on $\|u\|_{2 s_{0}+5}$, bounded as $u$ goes to zero. By an explicit computation, recalling (4.69) one gets $\left\{(1+\mathcal{L})^{\gamma}, \mathcal{L}\right\}=0$. This implies the 4.73).

(iii) This item follows by applying Proposition 2.5 and using that $\left\{(1+\mathcal{L})^{-\gamma},(1+\mathcal{L})^{\gamma}\right\}=0$. The bound (4.74) follows by (2.13) (with $\left.a \rightsquigarrow(1+\mathcal{L})^{-\gamma}, b \rightsquigarrow(1+\mathcal{L})^{\gamma}\right)$, (2.16), (4.71) and (2.28). The (4.75) follows by (2.31) using the (4.70).

(iv) To prove this item we use Neumann series. We define (using item (iii))

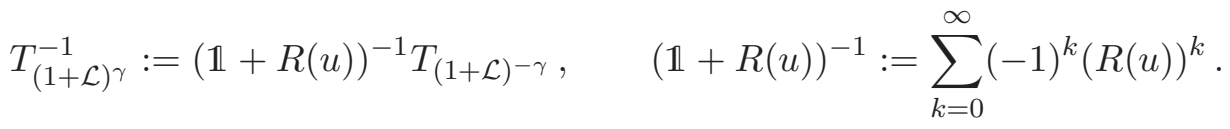

Using (4.75) and taking R sufficiently large one can check that

$$
\left\|(\mathbb{1}+R(u))^{-1} h\right\|_{H^{s}} \lesssim\|h\|_{H^{s}}\left(1+C\|u\|_{H^{2 s_{0}+5}}\right),
$$

for some $C>0$ depending on $\|u\|_{H^{2 s_{0}+5}}$, bounded as $u$ goes to zero. The bound above together with (4.72) implies the (4.76).

$(v)$ By (4.5) we have

$$
\partial_{t} \tilde{a}_{2, \mathrm{R}}^{+}=\frac{1}{2 \lambda_{2}}\left(2\left(1+\widetilde{a}_{2, \mathrm{R}}\right) \partial_{t} \widetilde{a}_{2, \mathrm{R}}-\partial_{t} \widetilde{b}_{2, \mathrm{R}} \overline{\widetilde{b}_{2, \mathrm{R}}}-\widetilde{b}_{2, \mathrm{R}} \partial_{t} \overline{\widetilde{b}_{2, \mathrm{R}}}\right) .
$$

Moreover, recalling (4.4), 3.5), the hypotheses of Lemma 2.13 are satisfied. Therefore, using (2.81) and (4.1), we deduce

$$
\left|\partial_{t} \widetilde{a}_{2, \mathrm{R}}\right|_{\mathcal{N}_{s_{0}}^{0}} \lesssim_{\mathrm{r}} C\|u\|_{H^{2 s_{0}+3}}
$$

Similarly one can prove the same estimate for $\widetilde{b}_{2}$. Hence the (4.77) follows. The (4.77) and (2.16) imply the (4.78).

(vi) Item (vi) follows by (2.11) since $\mathcal{L}, \mathcal{L}^{-1}$ are real valued.

In the following we shall construct the energy norm. By using this norm we are able to achieve the energy estimates on the previously diagonalized system. This energy norm is equivalent to the Sobolev one. For $s \in \mathbb{R}, s \geq 2 s_{0}+7$ we define

$$
w_{\gamma}:=T_{(1+\mathcal{L})^{\gamma}} w, \quad W_{\gamma}:=\left[\frac{w_{\gamma}}{w_{\gamma}}\right]=T_{(1+\mathcal{L})^{\gamma}} \mathbb{1} W, \quad W=\left[\frac{w}{w}\right], \quad \gamma:=\frac{s}{2} .
$$

Lemma 4.9 (Equivalence of the energy norm). Assume (4.1). Then, for $\mathrm{R}>0$ large enough, one has

$$
\left\|w_{\gamma}\right\|_{L^{2}} \sim_{\mathrm{r}}\|w\|_{H^{s}}
$$

where $\mathrm{r}$ is given in (4.1).

Proof. It follows by using estimates (4.72), (4.76) and reasoning as in the proof of Lemma 4.7.

Notice that, by using Lemma 2.3 (see (2.20) ) and by (4.64), the (4.63) is equivalent to (recall (4.69)

$$
\partial_{t} w=\mathrm{i} T_{\mathcal{L}} w+\mathrm{i} T_{a_{1, \mathrm{R}}^{(1)}} w+\mathcal{Q}_{1}(u) v+\mathcal{Q}_{2}(u) \bar{v}, \quad W:=\left[\frac{w}{w}\right],
$$


where

$$
\begin{aligned}
& a_{1, \mathrm{R}}^{(1)}(x, \xi):=a_{1}^{(1)}(x, \xi) \mathcal{X}_{\mathrm{R}}(\xi), \\
& \left\|\mathcal{Q}_{i}(u) h\right\|_{H^{s}} \lesssim_{\mathrm{r}} C\|h\|_{H^{s}}\|u\|_{H^{s}}, \quad \forall h \in H^{s}\left(\mathbb{T}^{d} ; \mathbb{C}\right), i=1,2, \quad s \geq 2 s_{0}+7,
\end{aligned}
$$

for some constant $C>0$ depending on $\|u\|_{H^{s}}$, bounded as $u$ goes to zero.

Lemma 4.10. Recall 4.81. One has that the function $w_{\gamma}$ defined in 4.79) solves the problem

$$
\partial_{t} w_{\gamma}=\mathrm{i} T_{\mathcal{L}} w_{\gamma}+\mathrm{i} \mathcal{A}_{\gamma}(u) w_{\gamma}+\mathcal{B}_{\gamma}(u) w_{\gamma}+\mathcal{R}_{\gamma}(u)[V],
$$

where

$$
\mathcal{A}_{\gamma}(u):=T_{(1+\mathcal{L})^{\gamma}} T_{a_{1, \mathrm{R}}^{(1)}}\left(T_{(1+\mathcal{L})^{\gamma}}\right)^{-1}, \quad \mathcal{B}_{\gamma}(u):=T_{\partial_{t}(1+\mathcal{L})^{\gamma}}\left(T_{(1+\mathcal{L})^{\gamma}}\right)^{-1},
$$

and where $\mathcal{R}_{\gamma}$ satisfies

$$
\left\|\mathcal{R}_{\gamma}(u) V\right\|_{L^{2}} \lesssim_{\mathrm{r}} C\|V\|_{H^{s}}\|u\|_{H^{s}},
$$

for some $C>0$ depending on $\|u\|_{H^{s}}$, bounded as u goes to zero.

Proof. By differentiating (4.79) and using (4.81) we get the (4.83) with $\mathcal{A}_{\gamma}(u), \mathcal{B}_{\gamma}(u)$ as in (4.84) up to a remainder $\mathcal{R}_{\gamma}$. The estimate (4.85) follows by (4.73), (4.55), (4.13), (4.72) and (4.82).

Proof of Theorem 4.1. We estimate the $L^{2}$-norm of $w_{\gamma}$ satisfying (4.83). Recalling (2.5), we have

$$
\partial_{t}\left\|w_{\gamma}\right\|_{L^{2}}^{2} \sim \operatorname{Re}\left(\mathrm{i}_{\gamma}(u) w_{\gamma}, w_{\gamma}\right)_{L^{2}}+\operatorname{Re}\left(\mathcal{B}_{\gamma}(u) w_{\gamma}, w_{\gamma}\right)_{L^{2}}+\operatorname{Re}\left(\mathcal{R}_{\gamma}(u) V, w_{\gamma}\right)_{L^{2}},
$$

where we have used that $\operatorname{Re}\left(\mathrm{i} T_{\mathcal{L}} w_{\gamma}, w_{\gamma}\right)_{L^{2}}=0$ thanks to item $(v i)$ of Lemma 4.8 . We analyze each summand separately. First of all we note that

$$
\left\|\mathcal{B}_{\gamma}(u) w_{\gamma}\right\|_{L^{2}} \stackrel{\sqrt[4.78,4.76]{\lesssim}}{\lesssim} C\|u\|_{H^{2 s_{0}+5}}\left\|w_{\gamma}\right\|_{L^{2}}
$$

for some constant $C>0$ depending on $\|u\|_{H^{2 s_{0}+5}}$, bounded as $u$ goes to zero. Hence, by Cauchy-Swartz inequality, we obtain

Using (4.85) we obtain

$$
\operatorname{Re}\left(\mathcal{B}_{\gamma}(u) w_{\gamma}, w_{\gamma}\right)_{L^{2}} \lesssim_{\Upsilon} C\|u\|_{H^{2 s_{0}+5}}\left\|w_{\gamma}\right\|_{L^{2}}^{2} .
$$

$$
\operatorname{Re}\left(\mathcal{R}_{\gamma}(u) V, w_{\gamma}\right)_{L^{2}} \lesssim_{\mathrm{r}} C\|u\|_{H^{s}}\left\|w_{\gamma}\right\|_{L^{2}}\|V\|_{H^{s}},
$$

for $s \geq 2 s_{0}+7$ and for some $C>0$ depending on $\|u\|_{H^{s}}$, bounded as $u$ goes to zero. We now study the most difficult term, i.e. the one depending on $\mathcal{A}_{\gamma}$. We write

$$
\mathcal{A}_{\gamma}(u)=T_{a_{1, \mathrm{R}}^{(1)}}+\mathcal{C}_{\gamma}(u), \quad \mathcal{C}_{\gamma}(u):=\left[T_{(1+\mathcal{L})^{\gamma}}, T_{a_{1, \mathrm{R}}^{(1)}}\right]\left(T_{(1+\mathcal{L})^{\gamma}}\right)^{-1} .
$$

By applying Proposition 2.5 and using estimates (4.23), (4.71), (2.28) and (4.76) we obtain

$$
\left\|\mathcal{C}_{\gamma}(u) w_{\gamma}\right\|_{L^{2}} \lesssim C\|u\|_{H^{2 s_{0}}+7}\left\|w_{\gamma}\right\|_{L^{2}}
$$

for some constant $C>0$ depending on $\|u\|_{H^{2 s_{0}+7}}$, bounded as $u$ goes to zero. Recall that the symbol $a_{1, \mathrm{R}}^{(1)}$ is real valued (see (4.21) ), then the operator $T_{a_{1, \mathrm{R}}^{(1)}}$ is self-adjoint w.r.t. the scalar product (2.5). As a consequence we have

$$
\operatorname{Re}\left(\mathrm{i} \mathcal{A}_{\gamma}(u) w_{\gamma}, w_{\gamma}\right)_{L^{2}} \stackrel{4.89}{=} \operatorname{Re}\left(\mathrm{i} \mathcal{C}_{\gamma}(u) w_{\gamma}, w_{\gamma}\right)_{L^{2}} \stackrel{\text { (4.90) }}{\lesssim} C\left\|w_{\gamma}\right\|_{L^{2}}^{2}\|u\|_{H^{2 s_{0}+7}}
$$

By (4.86), 4.87), (4.88) and 4.91) we get

$$
\partial_{t}\left\|w_{\gamma}\right\|_{L^{2}}^{2} \lesssim_{\Upsilon} C\left\|w_{\gamma}\right\|_{L^{2}}^{2}\|u\|_{H^{2 s_{0}+7}}+C\|u\|_{H^{s}}\left\|w_{\gamma}\right\|_{L^{2}}\|V\|_{H^{s}} .
$$

By 4.80, 4.68) the 4.92) becomes

$$
\partial_{t}\left\|w_{\gamma}\right\|_{L^{2}}^{2} \lesssim_{\Upsilon} C\|u\|_{H^{s}}\|v\|_{H^{s}}^{2} \quad \Rightarrow \quad\left\|w_{\gamma}\right\|_{L^{2}}^{2} \leq\left\|w_{\gamma}(0)\right\|_{L^{2}}^{2}+\int_{0}^{t} C\|u(\tau)\|_{H^{s}}\|v(\tau)\|_{H^{s}}^{2} d \tau,
$$

where $C>0$ depends on $\|u\|_{H^{s}}, r$. By using again the equivalences (4.80), (4.68) we get the (4.3). 
In the following we prove the existence of the solution of a linear problem of the form

$$
\left\{\begin{array}{l}
\dot{V}=\mathrm{i} E O p^{\mathrm{BW}}\left(|\xi|^{2} \mathbb{1}+A_{2}(x, \xi)+A_{1}(x, \xi)\right) V+R_{1}(U) V+R_{2}(U) U, \\
V(0)=V_{0}:=U(0),
\end{array}\right.
$$

where the para-differential part is assumed to be like in system (4.2) and $R_{2}(U) U$ has to be considered as a forcing term, the function $U$ satisfies (4.1) and the operators $R_{1}$ and $R_{2}$ are bounded.

Lemma 4.11. Let $s \geq 2(d+1)+7$. Consider the problem (4.93), assume (4.1) and that the matrices $A_{2}, A_{1}$ are like the ones in system (4.2). Assume moreover that $R_{i}$ are real-to-real and satisfy (3.10) for $i=1,2$. Then there exists a unique solution $V$ of $(4.2)$ in $L^{\infty}\left([0, T) ; H^{s}\left(\mathbb{T}^{d} ; \mathbb{C}^{2}\right)\right) \cap \operatorname{Lip}\left([0, T) ; H^{s-2}\left(\mathbb{T}^{d} ; \mathbb{C}^{2}\right)\right) \cap \mathcal{U}$, moreover it satsfies the following estimate

$$
\|V(t)\|_{H^{s}} \leq \mathrm{C}_{1} e^{\mathrm{C} T}\left((1+\mathrm{C} T)\left\|V_{0}\right\|_{H^{s}}+\mathrm{C} T\|U\|_{L^{\infty}\left([0, T) ; H^{s}\right)}\right),
$$

for positive constants $\mathrm{C}_{1}>0$ depending on $\mathrm{r}$ in (4.1) and $\mathrm{C}>0$ depending on $\|u\|_{H^{s}}, \mathrm{r}$ and bounded from above as $\|u\|_{H^{s}}$ goes to zero.

Proof. Let us consider first the case of the free equation, i.e. we assume for the moment $R_{1}(U) V=$ $R_{2}(U) U=0$. For any $\lambda \geq 1$ we consider the following localized matrix

$$
A^{\lambda}(x, \xi):=\left(A_{2}(x, \xi)+A_{1}(x, \xi)\right) \chi\left(\frac{\xi}{\lambda}\right),
$$

where $\chi$ is a cut-off function whose support is contained in the ball of center 0 and radius 1 . Note that the quantities $\left|A^{\lambda}\right|_{\mathcal{N}_{s}^{2}}$ and $\lambda^{-2}\left|A^{\lambda}\right|_{\mathcal{N}_{s}^{0}}$ (where we meant the semi-norm (2.6) of each entry) are uniformly bounded in $\lambda \geq 1$.

Let $V_{\lambda}$ be the solution of the following linear Schrödinger equation

$$
\left\{\begin{array}{l}
\dot{V}_{\lambda}=\mathrm{i} E O p^{\mathrm{BW}}\left(|\xi|^{2} \mathbb{1}+A^{\lambda}(x, \xi)\right) V_{\lambda} \\
V_{\lambda}(0)=V_{0} .
\end{array}\right.
$$

For initial data in $H^{s}$ the existence follows by the Duhamel formulation of the problem, a classical fixed point argument and using that $\lambda^{-2}\left|A^{\lambda}\right|_{\mathcal{N}_{s}^{0}}$ is uniformly bounded in $\lambda \geq 1$ (in other words $O p^{\mathrm{BW}}\left(A^{\lambda}\right)$ is a bounded perturbation with estimates depending on $\lambda$ ). The function $V_{\lambda}$ is continuous with values in $H^{s}$ with estimates which, a priori, depend on $\lambda$. We claim that the following estimate, with constants independent of $\lambda$, holds true:

$$
\left\|V_{\lambda}(t)\right\|_{H^{s}}^{2} \lesssim_{\mathrm{r}}\left\|V_{0}\right\|_{H^{s}}^{2}+\int_{0}^{t} C\|U(\sigma)\|_{H^{s}}\left\|V_{\lambda}(\sigma)\right\|_{H^{s}}^{2} d \sigma,
$$

for some $C>0$ depending $\|U\|_{H^{s}}, \mathrm{r}$ and bounded as $U$ goes to 0 . The proof of the claim follows the lines of Theorem 4.1 with $A(x, \xi)=A_{2}(x, \xi)+A_{1}(x, \xi)$ in (4.2) replaced by $A^{\lambda}$ in (4.95), we recall below the fundamental steps. One has to diagonalize the matrix $E\left(|\xi|^{2} \mathbb{1}+A^{\lambda}\right)$ as done in Propositions 4.4, 4.6 obtaining a new diagonal matrix of order two of the form

$$
\left(\begin{array}{cc}
|\xi|^{2}+a_{2}^{(1, \lambda)}(x, \xi)+a_{1}^{(1, \lambda)}(x, \xi) & 0 \\
0 & |\xi|^{2}+a_{2}^{(1, \lambda)}(x, \xi)+a_{1}^{(1, \lambda)}(x,-\xi)
\end{array}\right)
$$

where $\left|a_{j}^{(1, \lambda)}(x, \xi)\right|_{\mathcal{N}_{s}^{j}}$ are uniformly bounded in $\lambda$ for $j=1,2$. At this point, following Lemmata 4.9, 4.10, one obtains the energy estimate (4.96) by defining the new variable as in 4.79) with $\mathcal{L}$ replaced by $\mathcal{L}^{\lambda}:=|\xi|^{2}+a_{2}^{(1, \lambda)}(x, \xi)$. The constant in 4.96 is independent of $\lambda$ because $\left|\mathcal{L}^{\lambda}\right|_{\mathcal{N}_{s}^{2}}$ is uniformly bounded in $\lambda$.

As a consequence of (4.96) and Grönwall lemma we have

$$
\left\|V_{\lambda}(t)\right\|_{H^{s}}^{2} \lesssim_{\mathrm{r}}\left\|V_{0}\right\|_{H^{s}}^{2} \exp \left(\int_{0}^{t} C\|U(\sigma)\|_{H^{s}} d \sigma\right)
$$


which gives the uniform boundedness of the family in $L^{\infty}\left([0, T) ; H^{s}\left(\mathbb{T}^{d} ; \mathbb{C}^{2}\right)\right)$. This implies that the family is uniformly bounded in $C^{0}\left([0, T) ; H^{s}\left(\mathbb{T}^{d} ; \mathbb{C}^{2}\right)\right)$. Similarly, by using also (4.93), one proves that the family is uniformly Lipschitz continuous in the space $C^{0}\left([0, T) ; H^{s-2}\left(\mathbb{T}^{d} ; \mathbb{C}^{2}\right)\right)$, therefore one gets, up to subsequences, by Ascoli-Arzelà theorem, a limit $\Phi(t) U(0)$ in the latter space which is a solution of equation (4.93) with $R_{1}(U) V=R_{2}(U) U=0$. The limit $\Phi(t) U(0)$ is Lipschitz continuous with values in $H^{s-2}$. Moreover by using (4.3) and Grönwall lemma one obtains

$$
\left\|\Phi(t) V_{0}\right\|_{H^{s}}^{2} \lesssim_{\mathrm{r}}\left\|V_{0}\right\|_{H^{s}}^{2} \exp \left(\int_{0}^{t} C\|U(\sigma)\|_{H^{s}} d \sigma\right),
$$

i.e. $\left\|\Phi(t) V_{0}\right\|_{H^{s}} \lesssim_{\mathrm{r}}\left\|V_{0}\right\|_{H^{s}} e^{\mathrm{Ct}}$ for some $\mathrm{C}$ depending on $\|U\|_{H^{s}}, \mathrm{r}$ bounded as $U$ goes to 0 . The solution $\Phi(t) V_{0}$ is in $\mathcal{U}$ since $V_{0} \in \mathcal{U}$ and the matrix of symbols $A^{\lambda}$ in (4.95) is real-to-real.

To prove the existence of the solution in the case that $R_{1}(U) V$ and $R_{2}(U) U$ are non zero we use Picard iterates and we reason as follows. We define the operator

$$
T(W):=\Phi(t) V_{0}+\Phi(t) \int_{0}^{t}[\Phi(\sigma)]^{-1}\left(R_{1}(U) W(\sigma)+R_{2}(U) U(\sigma)\right) d \sigma
$$

and the sequence

$$
\left\{\begin{array}{l}
W_{0}=\Phi(t) U(0) \\
W_{n}=T\left(W_{n-1}\right) .
\end{array}\right.
$$

In this way we obtain that $\left\|W_{n+1}-W_{n}\right\|_{H^{s}} \leq \frac{(\mathrm{C} T)^{n}}{n !}\left\|W_{1}-W_{0}\right\|_{H^{s}}$. We find a fixed point for the operator $T$ as $V=\sum_{n=1}^{\infty} W_{n+1}-W_{n}+W_{0}$, the estimate (4.94) may be obtained by direct computation from the definition of the solution $W$.

Remark 4.12. If $R_{2}(U) U=0$ in the previous lemma, one gets the better estimate

$$
\|V(t)\|_{H^{s}} \leq \mathrm{C}_{1}(1+\mathrm{C} T) e^{T \mathrm{C}}\left\|V_{0}\right\|_{H^{s}} .
$$

\section{Proof of the main Theorem 1.2}

The proof of the Theorem 1.2 relies on the iterative scheme which is described below. We recall that, by Proposition 3.3, the equation (1.1) is equivalent to the para-differential system (3.8). We consider the following sequence of Cauchy problems

$$
\mathcal{P}_{1}=\left\{\begin{array}{l}
\partial_{t} U_{1}=\mathrm{i} E \Delta U_{1} \\
U_{1}(0, x)=\tilde{U}_{0}(x)
\end{array}\right.
$$

where $\tilde{U}_{0}(x)=\left(\tilde{u}_{0}, \overline{\tilde{u}}_{0}\right)$ is the initial condition of 1.1 , and we define by induction

$$
\mathcal{P}_{n}=\left\{\begin{array}{l}
\partial_{t} U_{n}=\mathrm{i} E O p^{\mathrm{BW}}\left(|\xi|^{2} \mathbb{1}+A_{2}\left(U_{n-1} ; x, \xi\right)+A_{1}\left(U_{n-1} ; x, \xi\right)\right) U_{n}+R\left(U_{n-1}\right) U_{n-1} \\
U_{n}(0, x)=\tilde{U}_{0}(x) .
\end{array}\right.
$$

In the following lemma we prove that the sequence is well defined, moreover the sequence of solutions $\left\{U_{n}\right\}_{n \in \mathbb{N}}$ is bounded in $H^{s}\left(\mathbb{T}^{d} ; \mathbb{C}^{2}\right)$ and converging in $H^{s-2}\left(\mathbb{T}^{d} ; \mathbb{C}^{2}\right)$.

Lemma 5.1. Fix $\tilde{U}_{0} \in H^{s}\left(\mathbb{T}^{d} ; \mathbb{C}^{2}\right) \cap \mathcal{U}$ such that $\left\|\tilde{U}_{0}\right\|_{H^{s}} \leq r$ with $s \geq 2(d+1)+9$, then there exists a time $T>0$ small enough such that the following holds true. For any $n \in \mathbb{N}$ the problem $\mathcal{P}_{n}$ admits a unique solution $U_{n}$ in $L^{\infty}\left([0, T) ; H^{s}\left(\mathbb{T}^{d} ; \mathbb{C}^{2}\right)\right) \cap \operatorname{Lip}\left([0, T) ; H^{s-2}\left(\mathbb{T}^{d} ; \mathbb{C}^{2}\right)\right)$. Moreover it satisfies the following conditions:

$(S 1)_{n}$ : There exists a constant $C$ depending on $s$ and $\left\|\tilde{U}_{0}\right\|_{H^{s-2}}$ such that for any $1 \leq m \leq n$ one has

$$
\left\|U_{m}\right\|_{L^{\infty}\left([0, T) ; H^{s}\right)} \leq \Theta, \quad \Theta:=C r .
$$

$(S 2)_{n}:$ For $1 \leq m \leq n$ one has $\left\|U_{m}-U_{m-1}\right\|_{L^{\infty}\left([0, T), H^{s-2}\right)} \leq 2^{-m+1}\left\|\tilde{U}_{0}\right\|_{H^{s-2}}$, where we have defined $U_{0}=0$. 
Proof. The proof of $(S 1)_{1}$ and $(S 2)_{1}$ is trivial, let us suppose that $(S 1)_{n-1}$ and $(S 2)_{n-1}$ hold true. We prove $(S 1)_{n}$ and $(S 2)_{n}$. We first note that $\left\|U_{n-1}\right\|_{L^{\infty} H^{s-2}}$ does not depend on $\Theta$. Indeed by using $(S 2)_{n-1}$ one proves that $\left\|U_{n-1}\right\|_{L^{\infty} H^{s-2}} \leq 2\left\|\tilde{U}_{0}\right\|_{H^{s-2}}$. Therefore Lemma 4.11 applies with $\mathrm{r}$ chosen large enough with respect to $\left\|\tilde{U}_{0}\right\|_{H^{s-2}}$, and the constants $\mathrm{C}, \mathrm{C}_{1}$ therein depend on $\Theta$ and $\left\|\tilde{U}_{0}\right\|_{H^{s-2}}$ respectively. We have the estimate

$$
\left\|U_{n}(t)\right\|_{H^{s}} \leq \mathrm{C}_{1} e^{\mathrm{C} T}\left((1+\mathrm{C} T)\left\|\tilde{U}_{0}\right\|_{H^{s}}+T \mathrm{C}\left\|U_{n-1}\right\|_{L^{\infty} H^{s}}\right) .
$$

To prove the $(S 1)_{n}$ we need to impose the bound

$$
\mathrm{C}_{1} e^{\mathrm{C} T}\left((1+\mathrm{C} T)\left\|\tilde{U}_{0}\right\|_{H^{s}}+T \mathrm{C} \Theta\right) \leq \Theta,
$$

this is possible by choosing $\mathrm{C}_{1} T \mathrm{C} \leq 1 / 8$ and $8 \mathrm{C}_{1}\left\|\tilde{U}_{0}\right\|_{H^{s}} \leq \Theta / 2$.

Let us prove $(S 2)_{n}$. We use the notation $A(U ; x, \xi):=|\xi|^{2} \mathbb{1}+A_{2}(U ; x, \xi)+A_{1}(U ; x, \xi)$ and $V_{n}:=$ $U_{n}-U_{n-1}$. The function $V_{n}$ solves the equation

$$
\partial_{t} V_{n}=\mathrm{i} E O p^{\mathrm{BW}}\left(A\left(U_{n-1} ; x, \xi\right)\right) V_{n}+f_{n},
$$

where

$$
f_{n}=\mathrm{i} E O p^{\mathrm{BW}}\left(A\left(U_{n-1} ; x, \xi\right)-A\left(U_{n-2} ; x, \xi\right)\right) U_{n-1}+R\left(U_{n-1}\right) U_{n-1}-R\left(U_{n-2}\right) U_{n-2} .
$$

The equation (5.2) with $f_{n}=0$ admits a well-posed flow $\Phi(t)$ thanks to Lemma4.11, moreover it satisfies the (4.98). Therefore by Duhamel principle we have

$$
\left\|V_{n}\right\|_{H^{s-2}} \leq\left\|\Phi(t) \int_{0}^{t}(\Phi(\sigma))^{-1} f_{n}(\sigma) d \sigma\right\|_{H^{s-2}} \leq \mathrm{C}_{1}^{2}(1+\mathrm{C} T)^{2} e^{2 T \mathrm{C}} \int_{0}^{t}\left\|f_{n}(\sigma)\right\|_{H^{s-2}} d \sigma .
$$

Using the Lipschitz estimates on the matrices $A$ (which may be deduced by (2.81) and $R$ (see the bound (3.11), and the inductive hypothesis one proves that $\left\|f_{n}\right\|_{H^{s-2}} \leq \mathrm{C}_{2}\left\|V_{n-1}\right\|_{H^{s-2}}$ for a positive constant $\mathrm{C}_{2}$ depending on $\Theta$ and $s$. Therefore by induction, using the choice of $T$ done in (5.1), one obtains $\left\|V_{n}(t)\right\|_{H^{s-2}} \leq \frac{(K t)^{n}}{n !}\left\|\tilde{U}_{0}\right\|_{H^{s-2}}$ for some $K$ independent on $n$, depending on $C_{2}$ and hence on $\Theta$ and $s$. By choosing $T$ in such a way that $K T<1$ one obtains the $(S 2)_{n}$.

We are now in position to prove the Theorem 1.2

Proof of Theorem 1.2. Fix $s>2(d+1)+9$. We first prove the existence of a weak solution of the Cauchy problem, then we prove that it is actually continuous and unique, finally we prove the continuity of the solution map.

Weak solutions. From Proposition 3.3 we know that equation (1.1) is equivalent to 3.8 . We consider the sequence of problems $\mathcal{P}_{n}$ previously defined. From Lemma 5.1 we obtain a sequence of solutions $U_{n}$ which is bounded in $L^{\infty}\left([0, T) ; H^{s}\left(\mathbb{T}^{d} ; \mathbb{C}^{2}\right)\right)$, by a direct computation one proves also that the sequence $\partial_{t} U_{n}$ is bounded in $L^{\infty}\left([0, T) ; H^{s-2}\left(\mathbb{T}^{d} ; \mathbb{C}^{2}\right)\right)$. Thus we get a weak-* limit $U \in L^{\infty}\left([0, T) ; H^{s}\left(\mathbb{T}^{d} ; \mathbb{C}^{2}\right)\right) \cap$ $\operatorname{Lip}\left([0, T) ; H^{s-2}\left(\mathbb{T}^{d} ; \mathbb{C}^{2}\right)\right)$, satisfying

$$
\|U\|_{L^{\infty} H^{s}} \leq \Theta=C\left\|\tilde{U}_{0}\right\|_{H^{s}},
$$

where $C>0$ is some constant depending on $\left\|\tilde{U}_{0}\right\|_{H^{s-2}}$. In order to show that the limit $U$ solves the equation it is enough to prove that it solves it in the sense of distribution. One can check that

$$
\left\|O p^{\mathrm{BW}}(A(U ; x, \xi)) U+R(U) U-O p^{\mathrm{BW}}\left(A\left(U_{n-1} ; x, \xi\right)\right) U_{n}-R\left(U_{n-1}\right) U_{n-1}\right\|_{H^{s-4}}
$$

goes to zero when $n$ goes to $\infty$, this is a consequence of triangular inequality, Lipschitz estimates on the matrix $A$ and $R$ and Lemma5.1 (in particular the boundedness of $U_{n}$ in $H^{s}\left(\mathbb{T}^{d} ; \mathbb{C}^{2}\right)$ and the strong convergence in $\left.H^{s-2}\left(\mathbb{T}^{d} ; \mathbb{C}^{2}\right)\right)$.

Remark 5.2. The fact that the time of existence $T>0$ depends only on $\left\|\tilde{U}_{0}\right\|_{H^{s}}$ is a consequence of Lemma 5.1 One could show that, in the case of small initial conditions of size $0<\varepsilon<1$, the time $T$ would be of order $O\left(\varepsilon^{-1}\right)$. 
Strong solutions. In order to prove that $U$ is in the space $C^{0}\left([0, T) ; H^{s}\left(\mathbb{T}^{d} ; \mathbb{C}^{2}\right)\right)$ we show that it is the strong limit of function in $C^{0}\left([0, T) ; H^{s}\left(\mathbb{T}^{d} ; \mathbb{C}^{2}\right)\right)$. We consider the following smoothed version of the initial condition

$$
V_{0}^{N}(x):=S_{\leq N} V_{0}(x):=\left(1-S_{>N}\right) V_{0}(x):=\sum_{|k| \leq N}\left(V_{0}\right)_{k} e^{\mathrm{i} k \cdot x},
$$

and we define $U^{N}$ the solution of (3.8) with initial condition $V_{0}^{N}$. The $U^{N}$, since $V_{0}^{N}$ is $C^{\infty}$ (in particular $\left.H^{s+2}\right)$, are in $C^{0}\left([0, T) ; H^{s}\left(\mathbb{T}^{d} ; \mathbb{C}^{2}\right)\right)$. We shall prove that $U^{N}$ converges strongly to $U$. We fix $\sigma+2+\varepsilon \leq$ $s, \sigma \geq 2(d+1)+7, \varepsilon>0$ and write $W:=U-U^{N}$, then $W$ solves the following problem

$$
\begin{aligned}
\partial_{t} W & =\mathrm{i} E O p^{\mathrm{BW}}(A(U ; x, \xi)) W+R(U) W \\
& +\mathrm{i} E O p^{\mathrm{BW}}\left(A(U)-A\left(U^{N}\right)\right) U^{N}+\left(R(U)-R\left(U^{N}\right)\right) U^{N},
\end{aligned}
$$

and $W(0, x)=\left(V_{0}-V_{0}^{N}\right)(x)$. We first study the $\sigma$ norm of the solution $W$. If one considers only the first line of the equation above then by Lemma4.11 and Remark 4.12 we have the existence of a flow $\phi(t)$ such that

$$
\|\phi(t) W(0, x)\|_{H^{\sigma}} \leq \mathrm{C}_{1}(1+\mathrm{C} T) e^{\mathrm{C} T}\left\|V_{0}-V_{0}^{N}\right\|_{H^{\sigma}} .
$$

By using the Duhamel formulation of the problem and the Lipschitz estimates we obtain

$$
\|W(t)\|_{H^{\sigma}} \leq C_{1}\left\|V_{0}-V_{0}^{N}\right\|_{H^{\sigma}}+C_{1} \int_{0}^{t}\left[\|W\|_{H^{\sigma}}\left\|U^{N}\right\|_{H^{\sigma+2}}(\tau)+\|W\|_{H^{\sigma}}\left\|U^{N}\right\|_{H^{\sigma}}(\tau)\right] d \tau,
$$

where $C_{1}>0$ depends on $\|U\|_{H^{\sigma}}$ and $\left\|U^{N}\right\|_{H^{\sigma}}$ and it is bounded as $U$ goes to 0 . Note that, since $\sigma+2<s$, the sequence $U^{N}$ is uniformly bounded in $H^{\sigma+2}\left(\mathbb{T}^{d} ; \mathbb{C}^{2}\right)$. By Grönwall Lemma we deduce that $\|W(t)\|_{H^{\sigma}} \leq \mathrm{C}_{3}\left\|V_{0}-V_{0}^{N}\right\|_{H^{\sigma}}$ for $\mathrm{C}_{3}>0$. Reasoning analogously for the $H^{s}\left(\mathbb{T}^{d} ; \mathbb{C}^{2}\right)$ norm one obtains

$$
\|W(t)\|_{H^{s}} \leq C\left\|V_{0}-V_{0}^{N}\right\|_{H^{s}}+C \int_{0}^{t}\left[\|W\|_{H^{\sigma}}\left\|U^{N}\right\|_{H^{s+2}}(\tau)+\|W\|_{H^{s}}\left\|U^{N}\right\|_{H^{s}}(\tau)\right] d \tau,
$$

where $C>0$ depends on $\|U\|_{H^{s}}$ and $\left\|U^{N}\right\|_{H^{s}}$ and is bounded as $U$ goes to 0 . The only unbounded term in the r.h.s. of the latter inequality is $\left\|U^{N}\right\|_{H^{s+2}}$. To analyze this term one can argue as follows. First of all, thanks to (5.3), we have that $\left\|U^{N}\right\|_{H^{s+2}} \leq \mathrm{C}_{4}\left\|V_{0}^{N}\right\|_{H^{s+2}}$, where $\mathrm{C}_{4}$ depends only on $\left\|V_{0}^{N}\right\|_{H^{s}}$. At this point one wants to use the well known smoothing estimate $\left\|V_{0}^{N}\right\|_{H^{s+2}} \lesssim N^{2}\left\|V_{0}\right\|_{H^{s}}$. To control the loss $N^{2}$ we use the previous estimate we have made on the factor $\|W\|_{H^{\sigma}} \lesssim C\left\|V_{0}-V_{0}^{N}\right\|_{H^{\sigma}}$, which may be bounded from above by $N^{-2-\varepsilon}\left\|V_{0}\right\|_{H^{s}}$. By (5.6) we get

$$
\|W(t)\|_{H^{s}} \leq C\left\|V_{0}-V_{0}^{N}\right\|_{H^{s}}+C \int_{0}^{t}\left[N^{-\varepsilon}\left\|V_{0}\right\|_{H^{s}}^{2}+\|W\|_{H^{s}}\left\|U^{N}\right\|_{H^{s}}(\sigma)\right] d \sigma .
$$

Hence we are ready to use Grönwall inequality again and conclude the proof.

Uniqueness. Let $V_{1}$ and $V_{2}$ be two solution of (3.8) with initial condition $V_{0}$. The function $W=V_{1}-V_{2}$ solves the problem

$$
\begin{aligned}
\partial_{t} W & =\mathrm{i} E O p^{\mathrm{BW}}\left(A\left(V_{1} ; x, \xi\right)\right) W+R\left(V_{1}\right) W \\
& +\mathrm{i} E O p^{\mathrm{BW}}\left(A\left(V_{1}\right)-A\left(V_{2}\right)\right) V_{2}+\left(R\left(V_{1}\right)-R\left(V_{2}\right)\right) V_{2},
\end{aligned}
$$

with initial condition $W(0, x)=0$. Arguing as before one proves that $\|W(t)\|_{H^{s-2}}=0$ for almost every $t$ in $[0, T)$ if $T$ is small enough. More precisely one considers the first line of the equation and applies Lemma 4.11 and Remark 4.12 to obtain a flow of such an equation in $H^{s-2}\left(\mathbb{T}^{d} ; \mathbb{C}^{2}\right)$ with estimates. Then, by means of the Duhamel formulation of the problem, thanks to the fact that the initial condition is equal to zero, the estimates on the flow previously obtained and Lipschitz estimates, one obtains $\|W\|_{H^{s-2}} \leq \frac{1}{2}\|W\|_{H^{s-2}}$ if $T$ is small enough with respect to $\left\|V_{1}\right\|_{H^{s}}$ and $\left\|V_{2}\right\|_{H^{s}}$. Since $W$ is continuous in time we deduce that is equal to 0 everywhere. 
Continuity of the solution map. The strategy is similar to the one adopted in [2], [26]. Let $\left\{\widetilde{U}_{n}\right\}_{n \geq 1} \subset$ $H^{s}\left(\mathbb{T}^{d} ; \mathbb{C}^{2}\right)$ be a sequence strongly converging to $\widetilde{U}_{0}$ in $H^{s}\left(\mathbb{T}^{d} ; \mathbb{C}^{2}\right)$. Consider $U_{n}$ and $U_{0}$ the solutions of the problem (3.8) with initial conditions respectively $\widetilde{U}_{n}$ and $\widetilde{U}_{0}$. We want to prove that $U_{n}$ converges strongly to $U_{0}$ in $H^{s}\left(\mathbb{T}^{d} ; \mathbb{C}^{2}\right.$ ). Let $T>0$ be small enough and fix $\varepsilon>0$. For $N_{\varepsilon}>0$ (to be chosen) we define

$$
\widetilde{U}_{0, \varepsilon}:=S_{\leq N_{\varepsilon}} \widetilde{U}_{0}, \quad \widetilde{U}_{n, \varepsilon}:=S_{\leq N_{\varepsilon}} \widetilde{U}_{n}
$$

where $S_{\leq N_{\varepsilon}}$ is defined as in (5.4) and define $U_{n, \varepsilon}$ and $U_{0, \varepsilon}$ the solutions of (3.8) with initial conditions $\widetilde{U}_{n, \varepsilon}$ and $\widetilde{U}_{0, \varepsilon}$ respectively. We note that

$$
\left\|U_{n}-U_{0}\right\|_{H^{s}} \leq\left\|U_{n}-U_{n, \varepsilon}\right\|_{H^{s}}+\left\|U_{n, \varepsilon}-U_{0, \varepsilon}\right\|_{H^{s}}+\left\|U_{0, \varepsilon}-U_{0}\right\|_{H^{s}} .
$$

Let us consider the first summand in (5.8). Let $W:=U_{n}-U_{n, \varepsilon}$ then, arguing as done to obtain the (5.7), one proves

$$
\|W(t)\|_{H^{s}} \leq C\left\|\widetilde{U}_{n, \varepsilon}-\widetilde{U}_{n}\right\|_{H^{s}}+C \int_{0}^{t}\left[N_{\varepsilon}^{-\delta}\left\|\widetilde{U}_{n}\right\|_{H^{s}}^{2}+\|W\|_{H^{s}}\left\|U_{n}\right\|_{H^{s}}(\sigma)\right] d \sigma,
$$

where $\delta>0$ is a positive small number. Since $\widetilde{U}_{n}$ converges to $\widetilde{U}_{0}$ in $H^{s}\left(\mathbb{T}^{d} ; \mathbb{C}\right)$ we have, that for $n$ large enough, $\left\|\widetilde{U}_{n}\right\|_{H^{s}} \leq 2\left\|\widetilde{U}_{0}\right\|_{H^{s}}$. Then by (5.3) there exists a constant $\Theta$, independent of $n \in \mathbb{N}$, such that $\left\|U_{n}\right\|_{H^{s}} \leq \Theta\left\|\widetilde{U}_{0}\right\|_{H^{s}}$. By Grönwall inequality and taking $T>0$ small enough one gets

$$
\left\|U_{n}-U_{n, \varepsilon}\right\|_{H^{s}} \lesssim\left\|\widetilde{U}_{n, \varepsilon}-\widetilde{U}_{n}\right\|_{H^{s}}+N_{\varepsilon}^{-\delta} .
$$

Notice that

$$
\begin{aligned}
\left\|\widetilde{U}_{n, \varepsilon}-\widetilde{U}_{n}\right\|_{H^{s}} & =\left\|S_{>N_{\varepsilon}} \widetilde{U}_{n}\right\|_{H^{s}} \\
& \leq\left\|S_{>N_{\varepsilon}}\left(\widetilde{U}_{n}-\widetilde{U}_{0}\right)\right\|_{H^{s}}+\left\|S_{>N_{\varepsilon}} \tilde{U}_{0}\right\|_{H^{s}} \\
& \leq\left\|\widetilde{U}_{n}-\widetilde{U}_{0}\right\|_{H^{s}}+\left\|S_{>N_{\varepsilon}} \tilde{U}_{0}\right\|_{H^{s}} \leq \varepsilon / 6,
\end{aligned}
$$

where to obtain the last inequality we have chosen, independently, $n$ and $N_{\varepsilon}$ large enough. Then we deduce that the r.h.s. in (5.9) may be bounded by $\varepsilon / 3$ (up to choose a bigger $N_{\varepsilon}$ ). In the same way one may prove that the third summand in (5.8) is bounded by $\varepsilon / 3$ again by choosing $N_{\varepsilon}$ large enough. We now study the second summand in (5.8). Arguing as done to obtain the (5.6) we get

$$
\left\|U_{n, \varepsilon}-U_{0, \varepsilon}\right\|_{H^{s}} \lesssim\left\|\widetilde{U}_{n, \varepsilon}-\widetilde{U}_{0, \varepsilon}\right\|_{H^{s}}+\int_{0}^{t}\left\|U_{n, \varepsilon}-U_{0, \varepsilon}\right\|_{H^{s}}\left\|U_{0, \varepsilon}\right\|_{H^{s+2}}(\tau) d \tau .
$$

By Grönwall inequality and taking $T>0$ small enough we obtain

$$
\left\|U_{n, \varepsilon}-U_{0, \varepsilon}\right\|_{H^{s}} \lesssim\left\|\widetilde{U}_{n, \varepsilon}-\widetilde{U}_{0, \varepsilon}\right\|_{H^{s}} \exp \left(N_{\varepsilon}^{2}\right) \lesssim\left\|\widetilde{U}_{n}-\widetilde{U}_{0}\right\|_{H^{s}} \exp \left(N_{\varepsilon}^{2}\right) .
$$

By taking $n \gg N_{\varepsilon}$, since $\widetilde{U}_{n} \rightarrow \widetilde{U}_{0}$ in $H^{s}$, we can conclude

$$
\left\|U_{n, \varepsilon}-U_{0, \varepsilon}\right\|_{H^{s}} \leq \varepsilon / 3 .
$$

By (5.8), we obtain $\left\|U_{n}-U_{0}\right\|_{H^{s}} \leq \varepsilon$, this implies the thesis.

\section{REFERENCES}

[1] P. Baldi, E. Haus, and R. Montalto. Controllability of quasi-linear Hamiltonian NLS equations. J. Differential Equations, 264(3):1789-1840, 2018.

[2] J. L. Bona and R. Smith. The initial-value problem for the Korteweg-de Vries equation. Philos. Trans. Roy. Soc. London Ser. A, 278(1287): 555-601, 1975.

[3] M. Berti and J.M. Delort. Almost global solutions of capillary-gravity water waves equations on the circle. UMI Lecture Notes, 2017. (awarded UMI book prize 2017).

[4] J. M. Bony. Calcul symbolique et propagation des singularités pour les équations aux dérivées partielle non linéaire. Ann. Sci. École Norm. Sup., 14:209-246, 1981.

[5] T. Cazenave. Semilinear Schrödinger Equations, volume 10. Courant lecture notes, 2003. 
[6] J.Y. Chemin and Salort D. Wellposedness of some quasi-linear Schrödinger equations. Science China math., 58(5):891-914, 2014.

[7] M. Christ. Illposedness of a Schrödinger equation with derivative nonlinearity. preprint.

[8] M. Colin and L. Jeanjean. Solutions for a quasilinear Schrödinger equation: a dual approach. Nonlinear Analysis, vol. 56(2), 213-226, 2004.

[9] P. Constantin and J.C. Saut. Local smoothing properties of Schrödinger equations. Indiana Univ. Math. J., 38(3):791-810, 1989.

[10] N. De Bouard, A. Hayashi and J.C. Saut. Global existence of small solutions to a relativistic nonlinear Schrödinger equation. Comm. Math. Phys., 189:73-105, 1997.

[11] N. De Bouard, A. Hayashi, P. Naumkin and J.C. Saut. Scattering problem and asymptotics for a relativistic nonlinear Schrödinger equation. Nonlinearity, 12(5):1415-1425, 1999.

[12] R. Feola and F. Iandoli. Local well-posedness for quasi-linear NLS with large Cauchy data on the circle. Annales de l'Institut Henri Poincare (C) Analyse non linéaire, 36(1):119-164, 2018.

[13] R. Feola and F. Iandoli. Long time existence for fully nonlinear NLS with small Cauchy data on the circle. Ann. Sc. Norm. Super. Pisa Cl. Sci., 22(5): 109-182, 2021.

[14] R. Feola and F. Iandoli. A non-linear Egorov theorem and Poincaré-Birkhoff normal forms for quasi-linear pdes on the circle. preprint, arXiv:2002.12448, 2020.

[15] R. Feola, B. Grébert and F. Iandoli. Long time solutions for quasi-linear Hamiltonian perturbations of Schrödinger and KleinGordon equations on tori. Preprint, arXiv:2009.07553, 2020.

[16] M. Goldman and M.V. Porkolab. Upper hybrid solitons and oscillating two-stream instabilities. Physics of Fluids, 19:872-881, 1976.

[17] R.W. Hasse. A general method for the solution of nonlinear soliton and kink Schrödinger equations. Z. Physik B, 3:83-87, 1980.

[18] T. Kato. Spectral Theory and Differential Equations. Lecture Notes in Mathematics, (eds.) Everitt, W. N., volume 448, chapter "Quasi-linear equations evolutions, with applications to partial differential equations". Springer, Berlin, Heidelberg, 1975.

[19] C. E. Kenig, G. Ponce, and L. Vega. Small solutions to nonlinear Schrödinger equations. Ann. I. H. Poincaré (C) Anal. Non Linéaire, 10(3):255-288, 1993.

[20] C. E. Kenig, G. Ponce, and L. Vega. Smoothing effect and local existence theory for the generalized nonlinear Schrödinger equations. Invent. Math., 134:489-545, 1998.

[21] C. E. Kenig, G. Ponce, and L. Vega. The Cauchy problem for quasi-linear Schrödinger equations. Invent. Math., 158:343-388, 2004.

[22] A.M. Litvak and A.G. Sergeev. One dimensional collapse of plasma waves. JETP, Letters, 194:517-520, 1978.

[23] V.K. Makhankov and V.G. Fedyanin. Non-linear effects in quasi-one- dimensional models of condensed matter theory. Physics reports, 104: 1-86, 1984.

[24] J. Marzuola, J. Metcalfe, and D. Tataru. Quasilinear Schrödinger equations I: Small data and quadratic interactions. Adv. Math., 231(2):1151-1172, 2012.

[25] J. Marzuola, J. Metcalfe, and D. Tataru. Quasilinear Schrödinger equations II: Small data and cubic nonlinearities. Kyoto J. Math, 54(3):529-546, 2014.

[26] J. Marzuola, J. Metcalfe, and D. Tataru. Quasilinear Schrödinger equations III: large data and short time. preprint, arXiv:2001.01014, 2020.

[27] G. Métivier. Para-Differential Calculus and Applications to the Cauchy Problem for Nonlinear Systems, volume 5. Edizioni della Normale, 2008.

[28] L. Molinet, J. C. Saut, and N. Tzvetkov. Ill-posedness issues for the Benjamin-Ono and related equations. SIAM J. of Math. Anal., 33:982-988, 2001.

[29] J. Moser. Rapidly convergent iteration method and non-linear partial differential equations - i. Ann. Sc. Norm. Sup. Pisa, 20(2):265-315, 1966.

[30] M. Poppenberg. Smooth solutions for a class of fully nonlinear Schrödinger type equations. Nonlinear Anal., Theory Methods Appl., 45(6):723-741, 2001.

[31] P. Rabinowitz. Free vibrations for a semi-linear wave equation. Comm. Pure Appl. Math. 31, 31:31-68, 1978.

[32] M. Taylor. Tools for PDE. Amer. Math. Soc., 2007.

[33] N. Tzvetkov. Ill-posedness issues for nonlinear dispersive equations. preprint, arXiv:math/0411455, 2007.

Dipartimento di Matematica, Università degli Studi di Milano, Via Saldini 50, I-20133

Email address: roberto. feola@unimi.it

Laboratoire Jacques Louis Lions (Sorbonne Université), 5 Place Jussieu, Paris 75005.

Email address: felice.iandoli@sorbonne-universite.fr 